Review

\title{
Nutrition Prescription to Achieve Positive Outcomes in Chronic Kidney Disease: A Systematic Review
}

\author{
Susan Ash ${ }^{1, *}$, Katrina L. Campbell ${ }^{2}$, Jessica Bogard ${ }^{3}$ and Anna Millichamp ${ }^{3}$ \\ 1 Institute of Health and Biomedical Innovation, School of Exercise and Nutrition Sciences, \\ Queensland University of Technology, Brisbane 4059, Australia \\ 2 Princess Alexandra Hospital, Brisbane 4001, Australia; \\ E-Mail: Katrina_cambpell@health.qld.gov.au \\ 3 School of Exercise and Nutrition Sciences, Queensland University of Technology, Brisbane 4059, \\ Australia; E-Mails: Jessica.bogard@live.com (J.B.); a.spongberg@student.qut.edu.au (A.M.) \\ * Author to whom correspondence should be addressed; E-Mail: s.ash@qut.edu.au; \\ Tel.: +61-401-086-594; Fax: +61-731-383-690.
}

Received: 27 October 2013; in revised form: 31 December 2013 / Accepted: 7 January 2014 / Published: 22 January 2014

\begin{abstract}
In Chronic Kidney Disease (CKD), management of diet is important in prevention of disease progression and symptom management, however evidence on nutrition prescription is limited. Recent international CKD guidelines and literature was reviewed to address the following question "What is the appropriate nutrition prescription to achieve positive outcomes in adult patients with chronic kidney disease?" Databases included in the search were Medline and CINAHL using EBSCOhost search engine, Embase and the Cochrane Database of Systematic Reviews published from 2000 to 2009. International guidelines pertaining to nutrition prescription in CKD were also reviewed from 2000 to 2013. Three hundred and eleven papers and eight guidelines were reviewed by three reviewers. Evidence was graded as per the National Health and Medical Research Council of Australia criteria. The evidence from thirty six papers was tabulated under the following headings: protein, weight loss, enteral support, vitamin D, sodium, fat, fibre, oral nutrition supplements, nutrition counselling, including protein and phosphate, nutrients in peritoneal dialysis solution and intradialytic parenteral nutrition, and was compared to international guidelines. While more evidence based studies are warranted, the customary nutrition prescription remains satisfactory with the exception of Vitamin D and phosphate. In these two areas, additional research is urgently needed given the potential of adverse outcomes for the CKD patient.
\end{abstract}


Keywords: chronic kidney disease; dietetics; evidence based practice; diet therapy; nutrition prescription

\section{Introduction}

Chronic kidney disease (CKD) is a prevalent chronic condition and the incidence of End-Stage Renal Disease (ESRD) is expected to continue to climb in the coming decade [1]. CKD has significant health and lifestyle implications for those affected, including increased risk of cardiovascular disease [2], malnutrition [3] and is a public health burden particularly in those patients who progress to end stage renal failure (or ESRD) and require kidney replacement therapy (dialysis) or transplantation [4]. The health cost burden is disproportionate to the prevalence with $5 \%$ of the health budget in the United States being consumed by $1 \%$ of the population requiring renal replacement [5]. CKD poses a significant public health issue and optimal treatment and management of this disease is indicated [6].

In CKD, nutrition and diet play an important role both in prevention of disease progression and in symptom management. The Dietitians Association Australia's (DAA) Evidence based guidelines for the nutritional management of Chronic Kidney Disease (CKD) stages 1-5 [7] provide statements of evidence against clinical questions in line with the Nutrition Care Process (NCP) [8]. The guidelines are designed to be employed by dietitians in clinical practice as the basis of nutritional management of patients with $\mathrm{CKD}$ and are based on the nutrition component of several recognized international guidelines. The evidence used, dates from published guidelines to 2005, and some of these guidelines varied in the method of rating evidence. Since 2006, a number of new international guidelines have been published or revised using an agreed grading system [9] and together with new literature these need to be reflected in dietetic practice, specifically the nutrition intervention or prescription employed by clinical dietitians. This article reviews the evidence presented in recent international guidelines and literature that address the clinical question "What is the appropriate nutrition prescription to achieve positive outcomes in adult patients with chronic kidney disease?"

\section{Methods}

A systematic literature review of studies was designed to answer the clinical question. Databases included in the search were Medline and CINAHL using EBSCOhost search engine, Embase and the Cochrane Database of Systematic Reviews. MeSH terms for Medline and CINAHL were "kidney failure, chronic" AND "diet therapy" OR "nutrition intervention" and for Cochrane "kidney failure, chronic". M-tree headings in EMBASE were "chronic kidney disease" AND "diet therapy" and further derivatives of diet therapy such as protein, phosphate. Results were limited to those published from 2000 to 2009, papers reported in the English language and studies involving adult humans. Articles were excluded if they were not reported in full or if they were presented as tutorials, editorials, news, letters or comments. Articles were also excluded if they were included within any systematic reviews or meta-analyses retrieved. The research aims and outcome measures reported on were used to assess applicability of the studies. Reference lists of retrieved papers were also reviewed and studies 
included where relevant. Nutritional management of acute renal disease, transplantation and nephrotic syndrome were not included in this review.

In addition to this systematic literature search, hand searches of recognised international guidelines published since 2006 and pertaining to nutrition were conducted. These included:

- European Renal Association/European Dietitian and Transplant Nurses Association ERA/EDTNA European Best Practice Guideline on Nutrition, 2007 [10],

- Guidelines for the management of chronic kidney disease by the Canadian Medical Association, 2008 [11],

- Diagnosis and management of chronic kidney disease: A national clinical guideline by the Scottish Intercollegiate Guidelines Network, 2008 [12],

- National Institute for Health and Clinical Excellence in the United Kingdom Chronic kidney disease: national clinical guideline for early identification and management in adults in primary and secondary care, 2008 [13],

- Caring for Australians with Renal Impairment (also known as CARI guidelines), 2013 [14-16],

- Kidney Disease: Improving Global Outcomes (KDIGO): Clinical practice guideline for the evaluation and management of Chronic Kidney Disease, 2012 [5,17],

- American Dietetic Association Chronic Kidney Disease Evidence-Based Nutrition Practice Guideline, 2010 [18],

- British Dietetic Association Evidence-based guidelines for the protein requirements of adults undergoing maintenance haemodialysis or peritoneal dialysis, 2013 [19].

Papers obtained through the literature search were categorized according to the aspect of nutrition prescription addressed in the research question, for example protein, phosphate, fat, vitamin $\mathrm{D}$, oral nutrition support. The strength of evidence of these papers was then assessed by three independent reviewers and categorized according to recommendations from the National Health and Medical Research Council (NHMRC) evidence hierarchy [20]. The NHMRC grades the level of evidence from I, a systematic review of all relevant randomized controlled trials to IV evidence obtained from case series. An overall grading of evidence is provided by NHMRC whereby level of evidence, consistency across studies, clinical impact and generalisability is also assessed from A, where the body of evidence can be trusted to guide practice to $\mathrm{D}$ were the body of evidence is weak and recommendations should be applied with caution. This system has been recognized as equivalent to the Kidney Disease Improving Global Outcomes (KDIGO) criteria [21].

In a similar fashion, evidence statements from international guidelines were then grouped according to aspects of the nutrition prescription addressed with their corresponding levels of evidence. The grading systems and definitions for levels of evidence and strength of practice recommendations used by the various institutions guidelines are compared in Appendix 1. Statements from the recent guidelines were then listed against each of the nutrition parameters defined above. 


\section{Results}

Database searches using the search terms described above yielded 325 individual papers. Following grading of the evidence quality and exclusion of papers of lower level evidence, 34 papers remained for inclusion in this review. Of these, five papers were systematic reviews, fourteen were randomized controlled trials, nine were prospective cohort or comparative studies with controls and six were interrupted time series or case series. Table 1 compares the systematic reviews of protein intake both in those with and without diabetes, weight management and enteral support on renal outcomes. Table 2 compares the evidence for the remaining studies according to nutrient parameters, such as protein, vitamin D, fats, sodium, fibre; or intervention, such as oral nutrition support, dietetic counseling, including phosphate, nutrients in peritoneal solution, intradialytic parenteral nutrition or percutaneous endoscopic gastrostomy feeding. Table 3 outlines statements from international guidelines against each of these parameters.

In Table 1, the systematic reviews of protein intake [22-24] indicate that in pre-dialysis, protein should be reduced to $0.6 \mathrm{~g} / \mathrm{kg}$ body weight/day or equivalent if using keto-analogues and diet combined. Reduction to this level resulted in a $32 \%$ reduction in renal deaths (RR $0.68,95 \%$ CI 0.55 to 0.8 , $p=0.0002$ ). For those with diabetes, both Types 1 and 2 , reducing protein is associated with moderate non-significant slowing in progression of diabetic nephropathy resulting in renal failure [24]. Protein intakes $<0.8 \mathrm{~g} / \mathrm{kg}$ body weight/day showed no compromise in anthropometry or biochemical indicators [23]. One systematic review was retrieved that examined weight loss interventions in CKD [25]. This review analysed 13 studies, two RCTs and 11 observational studies and found that only modest evidence exists to support the role of intentional weight loss on slowing CKD progression in mild-moderate CKD. A systematic review of enteral feeding in maintenance HD included 5 RCT and 13 non-RCT and concluded that enteral feeding, including oral nutrition support vs. routine care increased protein and energy intake and improved serum albumin by $0.23 \mathrm{~g} / \mathrm{dL}$ but there was insufficient data to examine the effect on clinical outcomes [26].

Table 2 outlines individual papers. There is evidence that for those patients with ESRD either Stage 4 or 5 , a very low protein diet $(0.3 \mathrm{~g} / \mathrm{kg} /$ day $)$ with added keto-analogues and adequate energy (35 kcal/day) can delay dialysis with no adverse effect on mortality [27]. Elderly patients with glomerular filtration rate (GFR) between 5 and $7 \mathrm{~mL} / \mathrm{min}$ on a similar diet, when compared to those on dialysis, had better outcomes with an improved survival of $3.6 \%(95 \% \mathrm{CI},-17$ to $+10 ; p=0.002)$ [28]. In dialysis, protein intakes of $>1.2 \mathrm{~g} / \mathrm{kg} /$ day resulted in significant increases in body mass index (BMI, $\left.\mathrm{kg} / \mathrm{m}^{2}\right)$ of $0.97(p<0.001)$ [29]. The association between protein intake and all-cause mortality and cardiovascular mortality amongst a large retrospective cohort, found that survival was best at protein intakes between 1.0 and $1.4 \mathrm{~g} / \mathrm{kg} /$ day and that intakes $<0.8 \mathrm{~g} / \mathrm{kg} /$ day and $>1.4 \mathrm{~g} / \mathrm{kg} /$ day were associated with increased mortality. However, this effect was diminished significantly when adjusted for malnutrition inflammation complex syndrome [30]. 
Table 1. Systematic reviews of nutrition interventions in patients with chronic kidney disease (CKD).

\begin{tabular}{|c|c|c|c|c|c|c|}
\hline Author & $\begin{array}{c}\text { Number of } \\
\text { Studies }\end{array}$ & Sample & Outcome Measures & Results & Conclusions & $\begin{array}{c}\text { Level of } \\
\text { Evidence [20] }\end{array}$ \\
\hline \multicolumn{7}{|c|}{ Protein (patients without diabetes) } \\
\hline Fouque [22] & $10 \mathrm{RCT}^{*} \mathrm{~s}$ & $\begin{array}{l}n=2000 \text { Pre-dialysis, } \\
\text { Stages } 3-5\end{array}$ & $\begin{array}{l}\text { Renal death (death of any cause, } \\
\text { requirement to start dialysis or } \\
\text { kidney transplant) }\end{array}$ & $\begin{array}{l}\text { RCTs or cross-over studies (if start date } \\
\text { allocated randomly). Protein intake } \\
(\geq 0.8 \mathrm{~g} / \mathrm{kg} / \text { day }) v s \text {. moderate }(0.6 \mathrm{~g} / \mathrm{kg} / \text { day) to } \\
\text { severe protein restriction }(0.3 \mathrm{~g} / \mathrm{kg} / \text { day }) \\
\text { regardless of supplementation with amino } \\
\text { acids or keto-acids. Participants with moderate } \\
\text { to severe } \mathrm{CKD} * \text { (as per GFR } * \text { serum } \\
\text { creatinine or creatinine clearance). }\end{array}$ & $\begin{array}{l}\text { A nutritional intervention that includes a reduction in } \\
\text { protein intake should be proposed to patients with } \\
\text { moderate } \mathrm{CRF} * \text {. Reducing protein intake overall } \\
\text { reduced renal deaths by about } 32 \%(p=0.0002) \text {. } \\
\text { Sub-analysis ( } 7 \text { studies) found that reduced protein } \\
\text { intakes between } 0.3 \text { and } 0.6 \mathrm{~g} / \mathrm{kg} / \text { day compared to } \\
\text { higher/free protein intakes resulted in a significant } \\
\text { reduction in renal deaths ( } 37 \%, p=0.0009) \text {.The } \\
\text { optimal level of protein intake cannot be determined } \\
\text { based on this review. }\end{array}$ & I \\
\hline Zarazaga [23] & $\begin{array}{l}26 \text { studies, } \\
\text { Including } 3 \\
\text { meta- } \\
\text { analyses } \\
\text { N.B. } \\
3 \text { studies } \\
\text { included } \\
\text { paediatric } \\
\text { patients }\end{array}$ & $\begin{array}{l}N=7155 \text { Dialysis }+ \\
\text { Pre-dialysis (Stages } \\
\text { not defined) }\end{array}$ & $\begin{array}{l}\text { Compliance with diet Mortality, } \\
\text { GFR, renal function } \\
\text { Anthropometry Biochemistry } \\
\text { (various factors that address } \\
\text { overall renal function) } \\
\text { Nutritional status }\end{array}$ & $\begin{array}{l}\text { Patients aged 2-65 years with chronic renal } \\
\text { failure in dialysis or pre-dialysis. Interventions } \\
\text { of nutritional support with amino acid or } \\
\text { keto-acid supplements with or without } \\
\text { restriction of protein intake. Protein } \\
\text { restricted to equivalent of } 0.6 \mathrm{~g} / \mathrm{kg} / \mathrm{day} \text {, } \\
\text { energy } 30-40 \mathrm{kcal} / \mathrm{day} \text { and } \\
\text { phosphate } 700-800 \mathrm{mg} / \text { day in interventions. }\end{array}$ & $\begin{array}{l}\text { Dietary protein should be restricted to } 0.4-0.6 \mathrm{~g} / \mathrm{kg} / \mathrm{day} \text {. } \\
\text { A protein intake of } 0.6 \mathrm{~g} / \mathrm{kg} / \text { day (comprising } \\
0.4 \mathrm{~g} / \mathrm{kg} / \mathrm{day}+0.2 \mathrm{~g} / \mathrm{kg} / \mathrm{day} \text { from supplements) } \\
\text { improves the course of renal function, nutritional status } \\
\text { and lipid profile, with good compliance. VLPD * and } \\
\mathrm{LPD} * \text { (using specific enteral supplements) should be } \\
\text { used by most patients in the early stages of CRF * to } \\
\text { slow progression of renal failure. For patients with CRF } \\
\text { on dialysis, prescription of a VLPD does not reduce } \\
\text { frequency of dialysis sessions. }\end{array}$ & I \\
\hline \multicolumn{7}{|c|}{ Protein (patients with diabetes) } \\
\hline Robertson [24] & $\begin{array}{l}12 \text { studies } \\
(9 \text { RCTs and } 3 \\
\text { before and after } \\
\text { studies) }\end{array}$ & $\begin{array}{l}n=585 \\
(\mathrm{~T} 1 \mathrm{DM}=322, \\
\mathrm{T} 2 \mathrm{DM}=263)\end{array}$ & $\begin{array}{l}\text { Compliance with low protein } \\
\text { diet Biochemistry (GFR) } \\
\text { All- cause mortality, ESRD * } \\
\text { Nutritional status, Health related } \\
\text { QOL *, Costs }\end{array}$ & $\begin{array}{l}\text { RCTs or before and after studies. Interventions } \\
\text { of reduced or modified protein intake } \geq 4 \text { months. } \\
\text { Participants of any age with type } 1 \text { or } 2 \mathrm{DM} * \\
\text { with nephropathy (UAER * } \geq 300 \mathrm{mg} / \text { day). }\end{array}$ & $\begin{array}{l}\text { Reducing protein intake is associated with a moderate, } \\
\text { non significant slowing in the progression of diabetic } \\
\text { nephropathy to renal failure. A specific recommendation } \\
\text { of the necessary protein level to achieve this outcome } \\
\text { is not possible. }\end{array}$ & I \\
\hline
\end{tabular}


Table 1. Cont

$\begin{array}{lllll} & & & \text { GFR, proteinuria, renal } \\ \text { 19 studies } & & \text { function, anthropometry/ } & \text { Patients with insulin dependent diabetes. } \\ \text { Zarazaga [23] } & \text { Including } & \text { nephropathy } & \text { nutritional status, } & \text { Interventions of nutritional support with amino } \\ & 1 \text { systematic } & \text { (Stages not defined) } & \begin{array}{l}\text { compliance with diet, } \\ \text { hyperglycemia, insulin }\end{array} & \text { acid or keto-acid supplements with or without } \\ & \text { review } & \text { restriction of protein intake } & \end{array}$

Protein restricted diets at least $<0.8-1 \mathrm{~g} / \mathrm{kg} /$ day is only recommended in Type $1 \mathrm{DM}$, showing reduction in hyperglycemia and decreased insulin requirements. Anthropometric parameters were preserved. LPD

(using specific enteral supplements) should be used by patients in the early stages of diabetic nephropathy to slow progression of renal failure. No specific protein intake levels are prescribed.

\section{Weight loss (patients with and without diabetes)}

$\begin{array}{lll} & & \\ & & \\ & 13 \text { studies } & (174 \text { non-surgical } \\ \text { Navaneethan }[25] & \text { (2 RCT and } & \text { interventions, } \\ & 11 \text { observational) }) & 346 \text { surgical } \\ & & \text { interventions }) \\ & \text { Stages } 1-4\end{array}$

\section{Renal function (GFR or} creatinine clearance, proteinuria).

Anthropometry (BMI *). Biochemistry (HbAlC *, serum lipids). Other (Blood Pressure)
Non-surgical weight loss did not elicit change in GFR or creatinine clearance, but was associated with a reduction in proteinuria, BMI, Systolic BP * and

Obese patients (BMI $\geq 30$ ). RCTs or observational studies of surgical or non-surgical weight loss interventions among patients with either existing CKD or obesity-related glomerular hyperfiltration. Follow up of $\geq 4$ weeks.

Total cholesterol. Surgical weight loss was associated with normalisation of GFR in glomerular hyperfiltration, significant reduction in BMI, proteinuria and systolic BP. Only modest evidence to support the role of intentional weight loss in slowing CKD progression in mild-moderate CKD

\section{Enteral support}

\begin{tabular}{|c|c|c|c|c|}
\hline Stratton [26] & $\begin{array}{l}18 \text { studies } \\
\text { ( } 5 \text { RCT and } \\
13 \text { non-RCT) }\end{array}$ & $\begin{array}{l}\text { Maintenance HD *, } \\
\text { Stage } 5\end{array}$ & $\begin{array}{l}\text { Clinical: QoL*, } \\
\text { Complications, mortality } \\
\text { Biochemical: albumin and } \\
\text { electrolyte levels } \\
\text { Nutritional: dietary intake, } \\
\text { anthropometry }\end{array}$ & $\begin{array}{l}\text { Multi-nutrient oral supplements and enteral } \\
\text { tube feeding which included nutrition support } \\
\text { (NS) with routine care; disease specific } \\
\text { formula with standard formulae; enteral } \\
\text { feeding with parenteral feeding. }\end{array}$ \\
\hline
\end{tabular}

Enteral feeding $v s$. routine care increased energy and protein intake and increased serum albumin concentration by $0.23 \mathrm{~g} / \mathrm{dL}(2.3 \mathrm{~g} / \mathrm{L}: 95 \% \mathrm{CI} * 0.037$ to $0.418 \mathrm{~g} / \mathrm{dL}$. There was insufficient data to examine the effect of this on clinical outcomes. Additional research required, especially comparing disease specific formulae with standard formulae

\footnotetext{
* LPD, Low protein diet; * CRF, Chronic Renal Failure; * GFR, Glomerular Filtration Rate; * RCT, Randomised Controlled Trial; * VLPD, Very low protein diet; * QOL, Quality of Life; * DM, Diabetes Mellitus;

* CKD Chronic Kidney Disease; * UAER, Urinary Albumin Excretion Rate; * BMI, Body Mass Index; * BP, Blood Pressure; * HbA1C, Glycosylated Haemoglobin; * HD, Haemodialysis;

* CI Confidence Interval.
} 
Table 2. Experimental and observational studies addressing various aspects of the nutrition prescription in CKD.

\begin{tabular}{|c|c|c|c|c|c|c|}
\hline Author & $\begin{array}{l}\text { Study Design } \\
\text { and Length }\end{array}$ & $\begin{array}{c}\text { Sample } \\
\text { Characteristics }\end{array}$ & Intervention & Outcomes & Results/Conclusions & $\begin{array}{c}\text { Level of } \\
\text { Evidence } \\
{[20]} \\
\end{array}$ \\
\hline \multicolumn{7}{|c|}{ Protein-experimental studies } \\
\hline Feiten $[31]$ & $\begin{array}{c}\mathrm{RCT} * \\
\text { (4 months) }\end{array}$ & $\begin{array}{l}n=24 \text { Pre-dialysis } \\
(\text { Stage } 4 \text { and } 5 \text { ) } \\
>18 \text { years }\end{array}$ & $\begin{array}{l}\text { Intervention: } \mathrm{VLPD} * \\
(0.3 \mathrm{~g} \text { vegetal protein } / \mathrm{kg} \mathrm{IBW} * / \text { day })+ \\
\mathrm{KA} * \text { Control: } \mathrm{LPD} \\
(0.6 \mathrm{~g} \text { protein } / \mathrm{kg} \text { IBW/day })\end{array}$ & $\begin{array}{l}\text { Nutrient intake \& compliance } \\
(3 \text { day food diary, normalised } \\
\text { protein appearance }(\mathrm{nNPA} *)) \text {. } \\
\text { Anthropometry }(\mathrm{BMI} * \\
\% \mathrm{TSF} *, \% \text { MAMC *, } \\
\text { LBMI *). Serum and urinary } \\
\text { urea, serum creatinine, ionised } \\
\text { calcium, bicarbonate, albumin, } \\
\text { iPTH, eGFR *) }\end{array}$ & $\begin{array}{l}\text { Nutritional status was maintained but compliance was poor in both } \\
\text { groups. Protein intake was underestimated by approximately } 28 \% \text { in } \\
\text { both groups when food records and nNPA were compared. Actual } \\
\text { protein intake of intervention group decreased significantly from } \\
0.9 \pm 0.24 \mathrm{~g} / \mathrm{kg} / \text { day to } 0.66 \pm 0.11 \mathrm{~g} / \mathrm{kg} / \text { day at } 4 \text { months }(p<0.05) \\
\text { while energy remained stable }(22.9 \mathrm{kcal} / \mathrm{kg} / \text { day in VLPD } * \text { and } \\
24 \mathrm{kcal} / \mathrm{kg} / \text { day in LPD. Serum urea nitrogen from } 61.4 \text { to } \\
43.6 \mathrm{mg} / \mathrm{dL}, p<0.001 \text {. Dietary } \mathrm{PO}_{4} * \text { decreased, with } \\
\text { improvements in } \mathrm{Ca} * \text { and } \mathrm{PTH} * \text { metabolism. }\end{array}$ & II \\
\hline Cianciaruso [32] & $\begin{array}{l}\text { Follow up data } \\
\text { from a RCT } \\
\text { (48 months) }\end{array}$ & $\begin{array}{l}n=423 \\
\text { Pre-dialysis } \\
\text { (stage } 4 \text { and 5) }\end{array}$ & $\begin{array}{l}\text { Intervention: LPD * } \\
(0.55 \mathrm{~g} / \mathrm{kg} / \text { dat }) \text { Control: MPD * } \\
(0.8 \mathrm{~g} / \mathrm{kg} / \text { day })\end{array}$ & $\begin{array}{l}\text { Protein energy malnutrition; } \\
\text { Progression to dialysis; } \\
\text { Mortality; Composite end point } \\
\text { (death or dialysis) }\end{array}$ & $\begin{array}{l}\text { Protein intakes were } 0.73 \pm 0.04 \mathrm{~g} / \mathrm{kg} / \text { day for LPD and } \\
0.9 \pm 0.06 \mathrm{~g} / \mathrm{kg} / \text { day for MPD. Unadjusted Cox survival analyses } \\
\text { were } 1.01(95 \% \mathrm{CI} * 0.57-1.79) 0.90(95 \% \mathrm{CI} 0.62-1.48) \text { and } \\
0.98(95 \% \mathrm{CI} 0.68-1.43) \text { respectively for death, progression to } \\
\text { dialysis or composite end point with no differences in outcome of } \\
\text { either intervention. }\end{array}$ & II \\
\hline Brunori $[28]$ & RCT (1 year) & $\begin{array}{l}n=112(\text { Stage } 5 \\
\text { GFR } 5-7 \mathrm{~mL} / \mathrm{min})\end{array}$ & $\begin{array}{l}\text { Intervention: LPD } \\
(0.3 \mathrm{~g} / \mathrm{kg} / \mathrm{day}, 35 \mathrm{kcal} / \mathrm{day}+ \\
\text { ketoacids, vitamins, minerals. } \\
\text { Control: Dialysis }\end{array}$ & $\begin{array}{l}\text { Mortality, hospitalization, } \\
\text { metabolic markers }\end{array}$ & $\begin{array}{l}\text { Median follow-up was } 26.5 \text { months (IQR *, } 40) \text {. Patients in diet } \\
\text { group spent median } 10.7 \text { months on VLPD (IQR *, } 11) .31 \text { deaths } \\
(55 \%) \text { in the dialysis group; } 28 \text { deaths }(50 \%) \text { in the diet group. } \\
\text { One-year observed survival rates at intention to treat } 83.7 \% \\
(95 \% \text { CI, } 74.5 \text { to } 94.0 \text { ) dialysis group versus } 87.3 \%(95 \% \mathrm{CI} \text {, } \\
78.9 \text { to } 96.5) \text { diet group; difference in survival }-3.6 \%(95 \% \mathrm{CI} \text {, } \\
-17 \text { to }-10 ; p=0.002) \text {. The hazard ratio for hospitalization was } \\
1.50 \text { for the dialysis group }(95 \% \mathrm{CI}, 1.11 \text { to } 2.01 ; p<0.01) \text {. }\end{array}$ & II \\
\hline
\end{tabular}


Table 2. Cont.

\begin{tabular}{|c|c|c|c|c|c|c|}
\hline \multicolumn{7}{|c|}{ Protein—observational studies } \\
\hline Vendrely [29] & $\begin{array}{l}\text { Comparative } \\
\text { study with } \\
\text { con-current } \\
\text { controls, } \\
12 \text { months }\end{array}$ & $\begin{array}{l}n=30 \text { Dialysis } \\
\left(\text { Stage } 5, \mathrm{HD}^{*}\right)\end{array}$ & $\begin{array}{l}\text { Intervention group: VLPD } \\
\text { ( } 0.3 \mathrm{~g} / \mathrm{kg} / \text { day supplemented with } \\
\text { essential amino acids, Calcium, } \\
\text { Iron and vitamins) prior to } \\
\text { initiation of } \mathrm{HD} \text {. Control: Less } \\
\text { restrictive diet ( } \sim 0.9 \mathrm{~g} / \mathrm{kg} / \text { day) } \\
\text { prior to initiation of } \mathrm{HD} \text {. }\end{array}$ & $\begin{array}{l}\text { Nutrient intake ( } 3 \text { day food } \\
\text { record every } 3 \text { months). } \\
\text { Anthropometry (BMI, body } \\
\text { composition by DEXA). Serum } \\
\text { albumin and } \\
\text { pre-albumin. }\end{array}$ & $\begin{array}{l}\text { Protein intake increased to }>1.2 \mathrm{~g} / \mathrm{kg} / \text { day, BMI increased by } \\
0.97 \pm 1.31 \mathrm{~kg} / \mathrm{m}^{2}, p<0.001 \text {, due to increased in fat mass } \\
2.36 \pm 2.94 \mathrm{~kg} / \mathrm{m}^{2}, p<0.001 \text { in both groups } 3 \text { months after } \\
\text { commencement of HD. No differences were observed between groups } \\
\text { for LBM, BMI, serum albumin or pre-albumin. }\end{array}$ & III-2 \\
\hline Kanazawa [33] & $\begin{array}{l}\text { Comparative } \\
\text { study with } \\
\text { concurrent } \\
\text { controls (not } \\
\text { randomised) }\end{array}$ & $\begin{array}{l}n=65 \\
\text { Pre-dialysis } \\
\text { (Stages 3-5) }\end{array}$ & $\begin{array}{l}\text { Case group: } \\
\text { Non-compliant on LPD } \\
(0.69 \mathrm{~g} / \mathrm{kg} / \text { day })>3 \text { months. } \\
\text { Control group: Compliant on LPD } \\
(0.69 \mathrm{~g} / \mathrm{kg} / \text { day })>3 \text { months }\end{array}$ & $\begin{array}{l}\text { Biochemistry }(\mathrm{GFR}, \text { serum } \\
\text { creatinine, BUN *, reciprocal of } \\
\text { serum creatinine). Dietary } \\
\text { compliance ( } 3 \text { day food records, } \\
\text { PCR *). Health related QOL * }\end{array}$ & $\begin{array}{l}\text { Change in mean GFR rate was lower in compliant group } \\
(-0.063 \pm 1.306 \text { compared to }-0.742 \pm 1.18, p<0.05 \text {. No difference } \\
\text { between groups for health-related QOL. }\end{array}$ & III-2 \\
\hline Shinaberger [30] & $\begin{array}{l}\text { Retrospective } \\
\text { cohort study. } \\
2 \text { years }\end{array}$ & $\begin{array}{l}n=53,933 \\
\text { Dialysis (Stage } 5, \\
\text { Maintenance HD) }\end{array}$ & $\begin{array}{l}\text { Historical review of maintenance } \\
\text { HD patients' protein intake } \\
\text { (measured by nPNA and } \\
\text { categorised into } 10 \text { increments) } \\
\text { \& mortality }\end{array}$ & $\begin{array}{l}\text { Protein intake ( measured by } \\
\text { nPNA) MICS * (malnutrition- } \\
\text { inflammation complex } \\
\text { syndrome) All-cause mortality } \\
\text { Cardiovascular mortality }\end{array}$ & $\begin{array}{l}\text { Hazard ratios were not significantly increased with nPNA between } \\
1-1.4 \mathrm{~g} / \mathrm{kg} / \text { day but increased to } 1.34 \text { ( } 95 \% \mathrm{CI} 1.23-1.46, p<0.0001 \text { ), } \\
\text { when levels were }<0.6 \text { or } \geq 1.4 \mathrm{~g} / \mathrm{kg} / \text { day. Protein intakes of }<0.8 \text { or } \\
>1.4 \mathrm{~g} / \mathrm{kg} / \text { day associated with greater mortality, even when adjusted } \\
\text { for MICS and case mix. Increasing protein intake of patients in } \\
\text { the } 0.8-1.2 \mathrm{~g} / \mathrm{kg} / \text { day protein range within the first } 6 \text { months, tended to } \\
\text { reduce mortality risk, whilst a decreased protein intake in the first } \\
6 \text { months, increased the risk. }\end{array}$ & III-3 \\
\hline Chauveau [27] & $\begin{array}{l}\text { Prospective } \\
\text { cohort study no } \\
\text { concurrent } \\
\text { controls, } 5 \text { years }\end{array}$ & $\begin{array}{l}n=203 \text { Predialysis } \\
(\text { Stage } 4-5)\end{array}$ & $\begin{array}{l}\text { VLPD }(0.3 \mathrm{~g} \text { protein } / \mathrm{kg} / \mathrm{day}, \\
35 \mathrm{kcal} / \mathrm{day}, \\
5-7 \mathrm{mg} \text { phosphate }+ \text { ketoacids }) \\
\text { for }>3 \text { months }\end{array}$ & $\begin{array}{l}\text { Mortality; Progression to dialysis } \\
\text { or transplant }\end{array}$ & $\begin{array}{l}\text { Mean duration of diet period } 33.1 \text { months (4-230). Overall survival } \\
\text { rate } 79 \% \text { and } 63 \% \text { at } 5 \text { and } 10 \text { year, respectively. } 102 \text { patients continued } \\
\text { with chronic dialysis during the entire follow-up, and } 101 \text { patients } \\
\text { were grafted at least once. No correlation between death and duration } \\
\text { of diet. }\end{array}$ & III-3 \\
\hline
\end{tabular}


Table 2. Cont.

\begin{tabular}{|c|c|c|c|c|c|c|}
\hline \multicolumn{7}{|c|}{ Vitamin D-experimental studies } \\
\hline Fishbane [34] & $\begin{array}{l}\text { RCT (double } \\
\text { blind, } 6 \text { months }\end{array}$ & $\begin{array}{l}n=61 \text { Pre-dialysis } \\
(\text { Stages } 1-4)\end{array}$ & $\begin{array}{l}\text { Intervention: oral } \\
\text { paricalcitol, } \\
1 \mu \mathrm{g} / \text { day Control: placebo }\end{array}$ & $\begin{array}{l}\text { Biochemistry (mean spot urinary } \\
\text { protein-creatinine ratio, serum intact } \\
\text { PTH, serum calcium, serum phosphorus, } \\
\text { urine creatinine) }\end{array}$ & $\begin{array}{l}\text { Significant decrease in proteinuria in paricalcitol group. Mean spot } \\
\text { urinary protein-creatinine ratios were }+2.9 \% \text { in controls and }-17.6 \% \\
\text { in the intervention group }(p=0.04) \text {. Serum iPTH } \downarrow \text { significantly in } \\
\text { intervention group }(p=0.01) .57 .6 \% \text { of paricalcitol group had a } \\
\text { more than } 10 \% \text { decline in proteinuria. Modest effect size noted as is } \\
\text { small study size. }\end{array}$ & II \\
\hline Agarwal [35] & $\begin{array}{l}\text { RCT (double } \\
\text { blind, } 24 \text { weeks }\end{array}$ & $\begin{array}{l}n=220 \\
\text { Pre-dialysis } \\
\text { (Stage 3-4) } \\
\text { Secondary } \\
\text { hyperparathyroidism }\end{array}$ & $\begin{array}{l}\text { Intervention: oral } \\
\text { paricalcitol } 9.5 \mu \mathrm{g} / \text { week } \\
\text { Control: placebo }\end{array}$ & Proteinuria & $\begin{array}{l}51 \% \text { intervention group compared to } 25 \% \text { control reduced } \\
\text { proteinuria (OR } 3.2,95 \% \text { CI } 1.5-6.9, p=0.004 \text { ). For those with } \\
\text { proteinuria and PTH suppression ( } 2 \text { consecutive } \geq 30 \% \text { decrease in } \\
\text { iPTH from baseline) proteinuria decreased } 53 \% \text { intervention } v s .0 \% \\
\text { in control. }\end{array}$ & II \\
\hline \multicolumn{7}{|c|}{ Vitamin D—observational studies } \\
\hline Wang [36] & $\begin{array}{c}\text { Cohort study } \\
\text { (prospective) } \\
3 \text { years }\end{array}$ & $\begin{array}{l}N=230 \text { Dialysis } \\
\left(\text { Stage } 5, \mathrm{PD}^{*}\right)\end{array}$ & $\begin{array}{l}\text { Serum Vitamin D } \\
(25(\mathrm{OH}) \mathrm{D}) \text { and clinical } \\
\text { outcomes (death, fatal } \\
\text { cardiovascular event, } \\
\text { non-fatal cardiovascular } \\
\text { event) }\end{array}$ & $\begin{array}{l}\text { Anthropometry }(\mathrm{BMI}) \text { Serum } 25(\mathrm{OH}) \mathrm{D} \text {, } \\
\text { eGFR echocardiography Nutritional } \\
\text { status (SGA *) Dialysis adequacy } \\
\text { All cause mortality Cardiovascular } \\
\text { events (fatal or non-fatal) }\end{array}$ & $\begin{array}{l}87 \% \text { of cohort were deficient or insufficient in } 25(\mathrm{OH}) \mathrm{D} \\
(<75 \mathrm{nmol} / \mathrm{L}) . \mathrm{Kaplan} \text { Meier estimates show a significantly greater } \\
\text { fatal or non-fatal } \mathrm{CV} * \text { event-free survival probability in patients } \\
\text { whose } 25(\mathrm{OH}) \mathrm{D}>\text { median } 45.7 \mathrm{nmol} / \mathrm{L} \text { than those with median } \\
\leq 45 \mathrm{nmol} / \mathrm{L}(p=0.004)\end{array}$ & III-2 \\
\hline \multicolumn{7}{|c|}{ Fats-experimental studies } \\
\hline Beavers [37] & $\begin{array}{l}\text { RCT (double } \\
\text { blind, permuted- } \\
\text { randomised), } \\
6 \text { months }\end{array}$ & $\begin{array}{l}n=69 \text { Dialysis } \\
(\text { Stage } 5, \mathrm{HD})\end{array}$ & $\begin{array}{l}\text { Intervention: daily } \\
\text { supplement of } 6 \mathrm{~g} n-3 \text { fatty } \\
\text { acids in the form of fish oil } \\
(160 \mathrm{mg} \text { EPA *, } 100 \mathrm{mg} \\
\text { DHA *) Control: } 6 \mathrm{~g} \text { daily } \\
\text { supplement corn oil }(n-6)\end{array}$ & $\begin{array}{l}\text { Biochemistry (total homocysteine) } \\
\text { Compliance: Pill counting (NB did not } \\
\text { use in vivo testing) }\end{array}$ & $\begin{array}{l}\text { Over the counter omega- } 3 \text { fatty acids at } 6 \mathrm{~g} \text { per day have no effect } \\
\text { on total homocysteine compared to a placebo. }\end{array}$ & II \\
\hline
\end{tabular}


Table 2. Cont.

\begin{tabular}{|c|c|c|c|c|c|c|}
\hline \multicolumn{7}{|c|}{ Fats-observational studies } \\
\hline Saltissi [38] & Case series, 14 weeks & $\begin{array}{l}n=75 \text { Dialysis } \\
\text { (Stage 5, HD and } \\
\text { PD) with } \\
\text { dyslipidaemia }\end{array}$ & $\begin{array}{l}\text { Dietary prescription: } \\
\text { Adjustment of "dialysis diet" } \\
\text { to bring in line with } \\
\text { Australian NHF * guidelines } \\
\text { to reduce lipid levels for } \\
\text { chronic PD and HD patients }\end{array}$ & $\begin{array}{l}\text { Anthropometry (BMI). Nutrient } \\
\text { intake: Dietary assessment and } \\
\text { computer analysis, } \\
\text { Biochemistry (total, HDL *, LDL *, } \\
\text { VLDL * cholesterol, TG *) }\end{array}$ & $\begin{array}{l}\text { In HD patients, decreased saturated fat and cholesterol intake was } \\
\text { associated with a decrease in total cholesterol }(p=0.007) \text { and } \\
\text { LDL cholesterol }(p<0.01) \text { but not in PD. Most dialysis patients } \\
\text { will require pharmacologic lipid lowering treatment for } \\
\text { adequate control. }\end{array}$ & IV \\
\hline \multicolumn{7}{|c|}{ Sodium and fluid-experimental studies } \\
\hline Vogt [39] & $\begin{array}{l}\text { RCT (double blind, } \\
\text { placebo controlled } \\
\text { crossover), } 36 \text { weeks }\end{array}$ & $\begin{array}{l}\text { Patients with } \\
\text { proteinuria } \\
\text { (various diagnoses) }\end{array}$ & $\begin{array}{l}\text { Intervention: Treatment } \\
\text { with placebo, Losartan, } \\
\text { Losartan }+\mathrm{HCT} * \text { whilst } \\
\text { randomised to either high } \\
\text { sodium ( } 200 \mathrm{mmol} / \text { day) } \\
\text { vs. low sodium } \\
\text { ( } 50 \mathrm{mmol} / \text { day }) \text { diet. }\end{array}$ & $\begin{array}{l}\text { Anthropometry (BMI). Biochemistry } \\
\text { (proteinuria, serum creatinine, urea, } \\
\text { cholesterol, triglycerides, total protein } \\
\text { and albumin). Other (urinary sodium } \\
\text { excretion, mean arterial pressure, } \\
\text { systolic and diastolic blood pressures) }\end{array}$ & $\begin{array}{l}\text { Baseline proteinuria was decreased by } 22 \% \text { by LSD * alone, } \\
\text { Losartan decreased proteinuria by } 30 \% \text {, Losartan }+ \text { LSD } \\
\text { decreased proteinuria by } 55 \% \text {. The combined addition of HCT } \\
\text { and low-sodium diet decreased proteinuria by } 70 \% \text { from baseline } \\
\text { (all } p<0.05 \text { ). Reductions in mean arterial pressure showed a } \\
\text { similar pattern (all } p<0.05 \text { ). A low sodium diet and HCT are } \\
\text { equally efficacious in reducing proteinuria and BP when added to } \\
\text { a regimen containing Losartan and especially seem to benefit } \\
\text { individuals in whom proteinuria is resistant to } \\
\text { Renin-Angiotensin-Aldosterone system blockade. Sodium } \\
\text { restriction exerted a modest but significant antiproteinuric effect. }\end{array}$ & II \\
\hline \multicolumn{7}{|c|}{ Sodium and fluid-observational studies } \\
\hline $\begin{array}{c}\text { Kayikcioglu } \\
{[40]}\end{array}$ & $\begin{array}{l}\text { Retrospective cross } \\
\text { sectional study } \\
\text { comparing } 2 \text { centres, } \\
\text { I year }\end{array}$ & $\begin{array}{l}n=394 \text { Stage } 5, \\
\text { HD. Centre A } \\
(n=190) \text {-salt } \\
\text { restriction. } \\
\text { Centre B } \\
(n=204) \text { - } \\
\text { hypertensive drugs }\end{array}$ & $\begin{array}{l}\text { Intervention: salt restricted } \\
\text { diet (5 g/day) and intensive } \\
\text { ultrafiltration to maintain } \\
\text { pre-dialysis B, } \\
P<140 / 90 \mathrm{mmHg} \text { without } \\
\text { antihypertensive medication. } \\
\text { Control: Hypertensive drugs }\end{array}$ & $\begin{array}{l}\text { Hypertensive drug use. } \\
\text { Weight and BP. Systolic } \\
\text { and diastolic function. Intradialytic } \\
\text { hypotension }\end{array}$ & $\begin{array}{l}\text { Antihypertensive drugs used in } 7 \% \text { Centre A and } 42 \% \text { in Centre } \\
\text { B }(p<0.01) \text {; Interdialytic weight gain was significantly lower in } \\
\text { Centre A ( } 2.29 \pm 0.83 \mathrm{~kg} \text { vs. } 3.31 \pm 1.12 \mathrm{~kg}, p<0.001) \text {. Mean } \\
\text { systolic and diastolic BP similar. Frequency of LV hypertrophy } \\
\text { was lower in Centre A ( } 74 \% \text { versus } 88 \%, p<0.001) \text {. } \\
\text { Intradialytic hypotension (hypotensive episodes } / 100 \text { patient } \\
\text { sessions) was more frequent in Centre B (11 versus } 27 \text {, } \\
p<0.01) \text {. }\end{array}$ & III-2 \\
\hline
\end{tabular}


Table 2. Cont.

\begin{tabular}{|c|c|c|c|c|}
\hline \multirow{5}{*}{$\begin{array}{c}\text { Boudville } \\
\text { [41] }\end{array}$} & \multirow{5}{*}{$\begin{array}{c}\text { Retrospective cohort, } \\
5 \text { years }\end{array}$} & \multirow{5}{*}{$\begin{array}{l}n=141 . \\
\text { (Stages } 4-5, \\
\text { including dialysis }+ \\
24 \mathrm{~h} \text { urine collection } \\
\text { for sodium) }\end{array}$} & \multicolumn{2}{|l|}{$24 \mathrm{~h}$ sodium excretion divided } \\
\hline & & & $\begin{array}{l}\text { into tertiles. Percentiles } 33.3 \\
\text { and } 66.6 \text { being } 114.0 \mathrm{mmol} / \mathrm{day}\end{array}$ & Hypertensive drug use. \\
\hline & & & Na. $(2.7 \mathrm{~g} /$ day $)$ and & BP control \\
\hline & & & $166.7 \mathrm{mmol} /$ day & \\
\hline & & & $\mathrm{Na}(4.0 \mathrm{~g} /$ day $)$, respectively & \\
\hline
\end{tabular}

Mean $( \pm \mathrm{SE})$ sodium excretion $145.7 \pm 4.7 \mathrm{mmol} /$ day

(3.5 g Na/day). Control of BP equivalent in all groups. Greater

no. antihypertensive agents with increased sodium excretion

$(2.00 \pm 0.16,2.61 \pm 0.20$, and $2.77 \pm 0.19$ medications,

respectively for each tertile; $p=0.01$ ). For those with

$\mathrm{GFR} \leq 15 \mathrm{~mL} / \mathrm{min}(n=77)$ medications used with increased

sodium excretion $1.69 \pm 0.19,2.52 \pm 0.27$, and $3.08 \pm 0.26$,

respectively; $p=0.001$. Multivariable analysis sodium

excretion $(p=0.00005)$ and age $(p=0.007)$ significantly

associated with use of antihypertensive medication.

\begin{tabular}{|c|c|c|c|c|c|c|}
\hline \multicolumn{7}{|c|}{ Fibre-experimental studies } \\
\hline Sutton [42] & $\begin{array}{c}\text { Interrupted time } \\
\text { series without parallel } \\
\text { control group }\end{array}$ & $\begin{array}{l}\text { Stage 1: } n=126 \\
\text { Stage 2: } 4 \text { weeks: } \\
n=23 \text { Stage 3: } \\
3 \text { weeks: } n=17 \\
\text { Dialysis (Stage 5, } \\
\text { PD) regularly using } \\
\text { laxatives }\end{array}$ & $\begin{array}{l}\text { Stage 1: Survey Stage 2: } \\
\text { laxative users replaced laxatives } \\
\text { with 6-12 g/day partially } \\
\text { hydrolysed guar gum supplement } \\
\text { Stage 3: dietary counselling to } \\
\text { support increased dietary fibre } \\
\text { intake of 6-12 g/day from foods }\end{array}$ & $\begin{array}{l}\text { Patient reported preference for } \\
\text { efficacy, ease of administration, } \\
\text { acceptability of taste and texture for } \\
\text { laxative, supplement or increased } \\
\text { dietary fibre.Self reported bowel } \\
\text { habits (Bristol stool chart) } \\
\text { Laxative use }\end{array}$ & $\begin{array}{l}\text { Of } 23 \text { patients involved in intervention, } 15 \text { thought the fibre } \\
\text { supplement provided best stool result and reduced side effects } \\
\text { and } 14 \text { preferred the supplement over laxative. No objective } \\
\text { data reported. Poor quality study, as reported outcomes were } \\
\text { not matched objectively against fibre intake. }\end{array}$ & IV \\
\hline \multicolumn{7}{|c|}{ Oral nutrition supplements—experimental studies } \\
\hline $\begin{array}{c}\text { Teixido- } \\
\text { Planas [43] }\end{array}$ & $\begin{array}{l}\text { Open RCT (multicentre), } \\
12 \text { months }\end{array}$ & $\begin{array}{l}n=65 . \text { Dialysis } \\
(\text { Stage } 5, \mathrm{PD})\end{array}$ & $\begin{array}{l}\text { Intervention: } 200 \mathrm{~mL} \\
(1.0 \mathrm{kcal} / \mathrm{mL}) \text { liquid protein } \\
\text { supplement daily in addition to } \\
\text { normal dietary intake. Control: } \\
\text { no protein supplement, usual } \\
\text { dietary intake }\end{array}$ & $\begin{array}{l}\text { Nutrient intake (3 day food record). } \\
\text { Anthropometry (BMI, skinfolds, } \\
\text { BSA *). Nutritional status (SGA). } \\
\text { Biochemistry (full blood count, serum } \\
\text { albumin, lymphocyte count, lipids, } \\
\text { urea, creatinine). Clinical (dialysis } \\
\text { adequacy, urinary and peritoneal } \\
\text { losses). Patient compliance (patient } \\
\text { report, family report, inventory check). }\end{array}$ & $\begin{array}{l}\text { Intention to treat analysis revealed a significant improvement } \\
\text { in the intervention group in lymphocyte count }(p<0.001) \text {, } \\
\text { weight }(p<0.03) \text {, TSF }(p<0.001) \text {, MAMC * }(p<0.005) \text {. } \\
\text { The supplement used was not suitable for long term use due } \\
\text { to a high rate of non-compliance and high dropout in the } \\
\text { intervention. Malnutrition assessed by SGA decreased from } \\
29 \% \text { in intervention to } 0 \% \text { and from } 33 \% \text { in controls to } 20 \% \text {. }\end{array}$ & II \\
\hline
\end{tabular}


Table 2. Cont.

$\begin{array}{llll} & & & \text { Intervention: } 200 \mathrm{~mL} \\ \text { Caglar [44] } & \text { Pilot prospective } & n=85 \text {. Dialysis } & (2.0 \mathrm{kcal} / \mathrm{mL} \text { ) liquid protein } \\ & \text { cohort study, } 9 \text { months, } & \text { (Stage } 5, & \text { supplement during dialysis } \\ & \text { with } 3 \text { months } & \text { treatment, } 3 \text { to } 9 \text { months. } \\ \text { baseline } & \text { malnutrition }) & \text { Control: standard nutritional } \\ & & \text { counselling, baseline to } \\ & & 3 \text { months }\end{array}$

Intervention: $200 \mathrm{~mL}$

supplement during dialysis

treatment, 3 to 9 months.

counselling, baseline to
ONS * improved nutritional parameters (significant increase in serum albumin $(3.33 \pm 0.32 \mathrm{~g} / \mathrm{dL}$ baseline to $3.65 \pm 0.26 \mathrm{~g} / \mathrm{dL}$ end 6 month intervention, $p<0.0001$ ), serum pre-albumin $(26.1 \pm 8.57 \mathrm{~g} / \mathrm{dL}$ baseline to $30.7 \pm 7.36 \mathrm{~g} / \mathrm{dL}$ end 6 month intervention, $p<0.0001)$ and SGA score $(4.94 \pm 1.23 \mathrm{~g} / \mathrm{dL}$ Anthropometry (BMI). Bioch (albumin, pre-albumin, transferrin). baseline to $5.64 \pm 0.90 \mathrm{~g} / \mathrm{dL}$ end 6 month intervention,

Nutritional status (SGA) $p<0.05)$ ). BMI and body weight increased non-significantly from baseline to end of intervention. Note: High non-compliance rate $(32 \%)$. Less than half of participants completed the study (46\%).
Frequency of moderate-severe malnutrition decreased $28 \%$ in intervention group ( $v s .6 \%$ in control group). Comparing baseline to 6 months, ONS significantly improved serum albumin $(2.64 \pm 0.35 v s .3 .05 \pm 0.72 \mathrm{~g} / \mathrm{dL})$ and energy intake
Intervention: nutritional counselling $+30 \mathrm{~g}$ oral GonzalesEspinoza [45] $n=28$. Dialysis (Stage 5, PD)

\section{egg-albumin protein} supplement of $22 \mathrm{~g}$ protein/day. Control: nutritional counselling.
Nutrient intake (24 h recall). Anthropometry (BMI, skin folds). Biochemistry (serum albumin, creatinine, lipids, nPNA, glucose, BUN *). Other (dialysis adequacy). Patient compliance (weighed inventory of supplement).
$(1331 \pm 342 v s .1872 \pm 698 \mathrm{kcal} / \mathrm{day})$ in the same group, $p<0.05$ and protein intake $(1.0 \pm 0.3 v s .1 .7 \pm 0.7 \mathrm{~g} / \mathrm{kg})$ and nPNA $(1.00 \pm 0.23$ vs. $1.18 \pm 0.35 \mathrm{~g} / \mathrm{kg} / \mathrm{day})$ within and between groups $(p<0.05)$ with a trend to increased intervention group. Multivariate analysis showed only serum albumin significantly predicted by ONS $(\beta 0.72,95 \% \mathrm{CI}$ $0.14-1.3, p=0.02)$ and $\%$ protein intake $(\beta-0.01,95 \% \mathrm{CI}$ $(0-0.02, p=0.05)$ and SGA significantly predicted by TSF (RR $0.79,95 \%$ CI $0.63-0.98, p=0.03$. Compliance was high at $90 \%$. 
Table 2. Cont.

\begin{tabular}{|c|c|c|c|c|c|c|}
\hline \multicolumn{7}{|c|}{ Nutritional Counselling_-intervention studies } \\
\hline $\begin{array}{l}\text { Campbell } \\
(2008)[46]\end{array}$ & RCT (12 weeks) & $\begin{array}{l}n=56 . \text { Pre-dialysis } \\
(\text { Stage } 4 \text { and } 5)\end{array}$ & $\begin{array}{l}\text { Intervention: Regular and } \\
\text { individualised dietary } \\
\text { counselling. Control: written } \\
\text { nutrition education material }\end{array}$ & $\begin{array}{l}\text { Nutrient intake (3 day food } \\
\text { record). Anthropometry (body } \\
\text { composition). Nutritional status } \\
\text { (SGA) }\end{array}$ & $\begin{array}{l}\text { Intervention group had a } 3.5 \%(95 \% \mathrm{CI}-2.1 \text { to } 9.1) \text {, less } \\
\text { decrease in body cell mass, } 17.7 \mathrm{~kJ} / \mathrm{kg} / \text { day }(95 \% \mathrm{CI} \\
8.2 \text { to } 27.2) \text { increase in energy intake, greater improvement in } \\
\text { SGA, all } p<0.01 \text { and no significant increase in protein intake. } \\
\text { Structured nutrition intervention had a greater effect on energy } \\
\text { and protein intake in women than men (interaction } p<0.001 \\
\text { for both). }\end{array}$ & II \\
\hline $\begin{array}{l}\text { Campbell } \\
\text { (2008) [47] }\end{array}$ & RCT (12 weeks) & $\begin{array}{l}n=53 . \text { Pre-dialysis } \\
\text { (Stage } 4 \text { and 5) }\end{array}$ & $\begin{array}{l}\text { Intervention: Regular and } \\
\text { individualised dietary } \\
\text { counselling. Control: written } \\
\text { nutrition education material } \\
\end{array}$ & $\begin{array}{l}\text { Nutritional status (PG-SGA *). } \\
\text { KDQoL * }\end{array}$ & $\begin{array}{l}\text { Intervention showed significant improvement in subscales of } \\
\text { KDQoL compared to nutritional status: symptoms } \\
7.1(0.1-14.1), p=0.047 \text {; cognitive functioning } \\
14.6(5.4-23.7), p=0.03 \text {; vitality } 12.0(4.6-19.5) p=0.002 \text {. }\end{array}$ & II \\
\hline \multicolumn{7}{|c|}{ Nutritional Counselling_-intervention studies } \\
\hline $\begin{array}{l}\text { Sullivan, } \\
\text { Sayre et al. } \\
2009[48]\end{array}$ & $\begin{array}{l}\text { Cluster RCT, } \\
14 \text { facilities, } 2 \text { shifts at } \\
12 \text { large centres } \\
\text { and } 1 \text { shift at } 2 \text { small } \\
\text { centres, } 3 \text { months }\end{array}$ & $\begin{array}{l}n=279 . \mathrm{HD} \text { (Stage 5). } \\
\text { Intervention } n=145 \text { : } \\
\text { Control } n=134\end{array}$ & $\begin{array}{l}\text { Intervention: education on avoiding } \\
\text { food with } \mathrm{PO}_{4} * \text { additives. Control: } \\
\text { Usual care. } 3 \text { month duration }\end{array}$ & Change in serum $\mathrm{PO}_{4}$ & $\begin{array}{l}\text { Intervention gp showed decrease in serum } \mathrm{PO}_{4} \text { of }-0.6 \mathrm{mg} / \mathrm{dL} \\
(95 \% \mathrm{CI}-1.0 \text { to }-0.1 \mathrm{mg} / \mathrm{dL}, p=0.03 \text { ). This change was not } \\
\text { explained by change in food knowledge score but intervention } \\
\text { group showed significant improvements in reading nutrition } \\
\text { facts label score } 9(95 \% \mathrm{CI} 1 \text { to } 17, p=0.04 \text { ) and food } \\
\text { ingredients list score } 22 \text { ( } 95 \% \text { CI } 15-30 p<0.001 \text { ). }\end{array}$ & II \\
\hline $\begin{array}{l}\text { Morey, } \\
\text { Walker } \\
\text { et al. [49] }\end{array}$ & $\mathrm{RCT}, 6$ months & $\begin{array}{l}n=67 \text { stable HD } \\
(\text { Stage } 5)\end{array}$ & $\begin{array}{l}\text { Intervention: Monthly dietetic } \\
\text { counselling to improve } \mathrm{PO}_{4} \\
\text { intake and binder adherence. } \\
\text { Control: } 6 \text { month counselling }\end{array}$ & $\begin{array}{l}\text { Change in serum } \mathrm{PO}_{4} \text {,controlling } \\
\text { for serum } \mathrm{PO}_{4} \text {, binder use and } \\
\text { alphacalcidrol at baseline }\end{array}$ & $\begin{array}{l}\text { Intervention group showed decrease in serum } \mathrm{PO}_{4} \text { at } 3 \text { months } \\
\text { approaching significance when controlled for } \\
\text { confounders }-0.253 \mathrm{mg} / \mathrm{dL}(95 \% \mathrm{CI}-0.513 \text { to } 0.007 \mathrm{mg} / \mathrm{dL} \text {, } \\
p=0.056 \text { ) compared to control however this difference } \\
\text { disappeared at } 6 \text { months. }\end{array}$ & II \\
\hline
\end{tabular}


Table 2. Cont.

\section{Nutritional Counselling—observational studies}

\section{Retrospective}

Campbell observational study,

(2009) [50] 2 years with 3 time

points $n=65$. Dialysis (Stage 5

maintenance $\mathrm{HD}$ )

Dietary interview (at least

every 6 months with intensive

follow up where required).
Nutrient intake (dietary interview)

Anthropometry (serum albumin and

potassium). Biochemistry. Nutritional

status (SGA)

Proportion of patients with malnutrition (as per SGA)

decreased from $14 \%$ to $3 \%$ after 2 years. Serum

albumin, potassium and dry weight remained stable.

Significant decrease in serum phosphate (mean $\pm \mathrm{SD}$

$1.8 \pm 0.5$ to $1.5 \pm 0.5 \mathrm{mmol} / \mathrm{L}, p=0.004)$. Energy

intake increased to $105 \mathrm{~kJ} / \mathrm{kg}$ from $102 \mathrm{~kJ} / \mathrm{kg}$ at

baseline $(p=0.001)$ and protein intake increased from

$1.14 \mathrm{~g} / \mathrm{kg} /$ day to $1.18 \mathrm{~g} / \mathrm{kg} /$ day $(p=0.022)$.

Under-reporting occurred in $30 \%-60 \%$ patients.

Nutrients in peritoneal dialysis solution—experimental studies

$\begin{array}{cll}\text { Tjong } & \text { Randomised } & \\ \text { (2005) [51] } & \text { cross-over study, } & n=8 . \text { Dialysis } \\ & 14 \text { days } & \text { (Stage 5, PD) }\end{array}$

Intervention: AAPD *

(plus glucose). Control: Standard

PD solution

Net protein balance (protein synthesis minus protein

breakdown) increased on AA PD in all patients (mean

\section{Nutrients in peritoneal dialysis solution-observational studies}

$\begin{array}{cll}\text { Sezer } & \begin{array}{l}\text { Prospective, open } \\ \text { (2006) [52] }\end{array} & \begin{array}{l}n=16 . \text { Dialysis } \\ \text { study, 3 months }\end{array} \\ & & \text { (Stage 5, PD) with } \\ & \text { hypoalbuminaemia }\end{array}$

Amino acid peritoneal dialysis (AAPD). 1 Dextrose peritoneal dialysate exchange/day replaced by a $2 \mathrm{~L}$ AAPD bag.
$0.21 \pm 0.12 \mu$ mol leucine $/ \mathrm{kg}$ per min; $p<0.001$ ). The

24 -h nitrogen balance changed by $0.96 \pm 1.21 \mathrm{~g} /$ day,

from $-0.60 \pm 2.38$ to $0.35 \pm 3.25 \mathrm{~g} /$ day $(p=0.061$

NS), improving in six patients.

Biochemistry (WBPT * $24 \mathrm{~h}$ nitrogen

balance)

Albumin improved $3.5 \pm 0.5 \mathrm{~g} / \mathrm{dL}$ to $4.1 \pm 0.4 \mathrm{~g} / \mathrm{dL}$

Anthropometry $(\mathrm{LBM} *)$. Biochemistry $\quad(p=0.003)$; HDL cholesterol level decreased from

(albumin, lipids). Nutritional status

(SGA)
$43.1 \pm 7.3 \mathrm{mg} / \mathrm{dL}$ to $37.8 \pm 6.0 \mathrm{mg} / \mathrm{dL}$ ( $p=0.02$ ), even

though other lipid parameters (total cholesterol,

triglyceride and LDL cholesterol) did not change. 
Table 2. Cont

\section{Intradialytic Parenteral Nutrition-experimental studies}

\begin{tabular}{|c|c|c|c|c|}
\hline $\begin{array}{c}\text { Pupim } \\
\text { (2004) [53] }\end{array}$ & $\begin{array}{l}\text { Randomised } \\
\text { prospective cross over } \\
\text { study }\end{array}$ & $\begin{array}{l}n=7 . \text { Dialysis } \\
(\text { Stage } 5, \mathrm{HD})\end{array}$ & IDPN * & $\begin{array}{l}\text { Biochemistry (albumin fractional } \\
\text { synthetic rate, WBPT *) }\end{array}$ \\
\hline $\begin{array}{c}\text { Pupim } \\
\text { (2006) [54] }\end{array}$ & $\begin{array}{l}\text { Randomised } \\
\text { prospective cross over } \\
\text { study }\end{array}$ & $\begin{array}{l}n=8 \text {. Dialysis } \\
\text { (Stage 5, HD) }\end{array}$ & $\begin{array}{l}\text { Intervention: IDPN or oral } \\
\text { nutritional supplement during } \\
\text { HD treatment. Control: normal } \\
\text { HD treatment }\end{array}$ & $\begin{array}{l}\text { Biochemistry (albumin, prealbumin, } \\
\text { transferrin, metabolic hormones, serum } \\
\text { protein, etc.) }\end{array}$ \\
\hline
\end{tabular}

Nutritional supplementation in the form of IDPN improves the hepatic synthesis of albumin $(16.2 \pm 1.5 \% /$ day $v$.

$12.8 \pm 1.7 \% /$ day, $p<0.05$ ) as a part of improvements in the whole body protein synthesis $(5.05 \pm 0.3 \mathrm{mg} / \mathrm{kg}$ fat-free mass $/ \mathrm{min} v s .3 .22 \pm 0.3 \mathrm{mg} / \mathrm{kg}$ fat-free mass $/ \min (p<0.05)$. Positive whole-body net balance during HD with both IDPN and ONS, $4.43 \pm 0.7$ and $5.71 \pm 1.2 \mathrm{mg} / \mathrm{kg}$ fat-free mass per $\mathrm{min}$, respectively, compared with control $(0.25 \pm 0.5 \mathrm{mg} / \mathrm{kg}$ fat-free mass per min; $p=0.002$ and $<0.001$ ) for IDPN versus control and ONS versus control, respectively. ONS resulted in persistent anabolic benefits in the post-HD phase for muscle protein metabolism, when anabolic benefits of IDPN dissipated.

Intradialytic Parenteral Nutrition—observational studies

\begin{tabular}{|c|c|c|c|c|c|c|}
\hline $\begin{array}{l}\text { Cherry } \\
(2002)[55]\end{array}$ & Case series, 12 months & $\begin{array}{l}n=24 . \text { Dialysis } \\
\text { (Stage 5, PD). } \\
\text { Malnourished, using } \\
\text { criteria }\end{array}$ & $\begin{array}{l}\text { Intervention: } 2 \text { formulations } \\
750 \mathrm{~mL} \text { and } 1000 \mathrm{~mL} \text { IDPN, } \\
\text { both } 925 \text { non protein calories, } \\
1000-\mathrm{mL} \text { formulation provided } \\
\text { an extra } 25 \mathrm{~g} \text { of protein. }\end{array}$ & $\begin{array}{l}\text { Anthropometry (dry body weight). } \\
\text { Biochemistry (serum albumin) }\end{array}$ & $\begin{array}{l}\text { Body weight increased from median } 46.8 \mathrm{~kg} \text { at baseline to } \\
47.5 \text { at } 6 \text { months and } 53.8 \text { at } 12 \text { months }(p<0.05, p<0.05 \text {, } \\
p<0.003 \text { respectively). Serum albumin levels increased from } \\
\text { median of } 27.5 \text { at baseline to } 31.0 \text { at } 3 \text { months }(p<0.05 \text { ) and } \\
30.5 \text { at } 12 \text { months in malnourished HD patients. Significant } \\
\text { attrition at } 9 \text { and } 12 \text { months }(n=16)\end{array}$ & IV \\
\hline \multicolumn{7}{|c|}{ Intradialytic Parenteral Nutrition—observational studies } \\
\hline $\begin{array}{l}\text { Joannidis } \\
\text { (2008) [56] }\end{array}$ & $\begin{array}{l}\text { Prospective cohort } \\
\text { study with matched } \\
\text { controls, } 6 \text { months }\end{array}$ & $\begin{array}{l}n=12 . \text { Dialysis } \\
\text { (Stage 5, PD) with } \\
\text { MICS. Controls had no } \\
\text { malnutrition }\end{array}$ & $\begin{array}{l}\text { Intervention: IDPN } 100 \mathrm{~mL} \\
\text { glucose } 60 \%, 100 \mathrm{~mL} \text { Elolipid } \\
20 \% \text { (soya bean oil } \\
100 \mathrm{~g} / 1000 \mathrm{~mL} \text {, glycerol } \\
25 \mathrm{~g} / 1000 \mathrm{~mL} \text {, egglecithin } \\
12 \mathrm{~g} / 1000 \mathrm{~mL} \\
\text { Control: usual dialysis }\end{array}$ & $\begin{array}{l}\text { Anthropometry (weight, BMI) } \\
\text { Biochemistry (lipids, inflammatory } \\
\text { markers }\end{array}$ & $\begin{array}{l}\text { Mean body weight increased from } 61.7 \pm 7.7 \text { to } 63.9 \pm 8.9 \mathrm{~kg} \\
(p=0.03) \text { and BMI increasred from } 21.9 \pm 3.4 \text { to } \\
22.8 \pm 3.9 \mathrm{~kg} / \mathrm{m}^{2}, p=0.03 \text {, compared to no change in control } \\
\text { group. } \mathrm{nPCR} \text { values differed significantly between patients at } \\
\text { baseline but no significant difference was observed at the } \\
\text { completion of the study for any other biochemical or } \\
\text { nutritional markers. }\end{array}$ & III-2 \\
\hline
\end{tabular}


Table 2. Cont

\begin{tabular}{|c|c|c|c|c|c|c|}
\hline $\begin{array}{c}\text { Korzets } \\
\text { (2008) [57] }\end{array}$ & $\begin{array}{l}\text { Prospective } \\
\text { observational case } \\
\text { series, } 1.5 \text { to } 17 \text { months }\end{array}$ & $\begin{array}{l}n=22 . \text { Dialysis } \\
\text { (Stage } 5, \mathrm{HD})\end{array}$ & $\begin{array}{l}\text { IDPN: Total E } 1174-1677 \mathrm{kcal} \\
\text { Amino acids } 10 \% 50-85 \mathrm{~g} \text {; } \\
\text { dextrose } 50 \% 125-185 \mathrm{~g} \text {; } \\
\text { Clinoleic } 20 \% 50-70 \mathrm{~g} \text {, } \\
\text { following major surgical or } \\
\text { medical illnesses }\end{array}$ & $\begin{array}{l}\text { Anthropometry. Biochemistry } \\
\text { (protein catabolic rate, } \\
\text { albumin, pre-albumin, } \\
\text { creatinine). Dialysis adequacy }\end{array}$ & $\begin{array}{l}\mathrm{nPCR} \text { increased from } 0.7 \pm 0.2 \text { to } 1.2 \pm 0.2 \mathrm{~g} \text { protein } / \mathrm{kg} / \text { day } \\
(p<0.0001) \text {; serum albumin increased from } 28 \pm 5 \mathrm{~g} / \mathrm{L} \text { to } 38 \pm 2 \mathrm{~g} / \mathrm{L} \\
(p<0.0001) \text {; serum pre-albumin levels increased from } 210 \pm 82 \text { to } \\
300 \pm 52 \mathrm{mg} / \mathrm{L} \text { ( } p<0.01 \text { and serum creatinine increased from } \\
504 \pm 195 \text { to } 672 \pm 186 \mu \mathrm{mol} / \mathrm{L}(p=0.016) \text {. Serum cholesterol } \\
\text { increased from } 3.5 \pm 1.4 \text { to } 4.4 \pm 1.4 \mathrm{mmol} / \mathrm{L}(p<0.0001) . \mathrm{Kt} / \mathrm{V} \text { levels } \\
\text { and weight did not change significantly during IDPN }(1.43 \pm 0.22 \text { to } \\
1.46 \pm 0.26) \text {. }\end{array}$ & IV \\
\hline \multicolumn{7}{|c|}{ Percutaneous Endoscopic Gastrostomy (PEG) feeding } \\
\hline $\begin{array}{c}\text { (Sayce } \\
\text { 2000) [58] }\end{array}$ & $\begin{array}{l}\text { Case series. Pre and } \\
\text { post intervention over } \\
3 \text { months }\end{array}$ & $\begin{array}{l}n=8 . \text { Dialysis (Stage } 5, \\
\text { HD). +malnutrition }\end{array}$ & $\begin{array}{l}\text { Various PEG feeding regimens; } \\
\text { E } 1983-7205 \mathrm{kcal} / \text { day; } \\
\text { Pro } 17-61 \mathrm{~g} / \text { day }\end{array}$ & $\begin{array}{l}\text { Anthropometry (weight, skin } \\
\text { folds). Biochemistry (albumin). } \\
\text { Cost (hospitalisations and } \\
\text { complications) }\end{array}$ & $\begin{array}{l}\text { Median dry weight increased from } 43 \text { to } 48.3 \mathrm{~kg}(p=0.012) \text {; BMI } \\
\left.\text { increased from } 16.4 \text { to } 18.8 \mathrm{~kg} / \mathrm{m}^{2} \text { ( } p=0.012\right) \text {; MUAC increase from } \\
20.2 \text { to } 24.8 \mathrm{~cm}(p=0.018) \text {; TSF increased from } 7.3 \text { to } 11.3 \mathrm{~mm} \\
\text { ( } p=0.046) \text {; MUAMC increased from } 17.7 \text { to } 19.8 \mathrm{~cm}(p=0.027) \text {; } \\
\text { Serum albumin increased from } 29.5 \text { to } 36.5 \mathrm{~g} / \mathrm{L}(p=0.011)\end{array}$ & IV \\
\hline
\end{tabular}

* CKD Chronic Kidney Disease; * HD Haemodialysis; * PD Peritoneal Dialysis; * GFR Glomerular Filtration Rate; * RCT Randomised Controlled Trial; * MPD Moderate protein diet; * LPD Low protein diet;

* VLPD Very low protein diet; * KA Keto-acids; * NHF National Heart Foundation; * LSD Low sodium diet; * HSD High sodium diet, * BUN Blood urea nitrogen; * QOL quality of life; * POM profile of mood states; * BSA body surface area; * iPTH intact parathyroid hormone; * AAPD Amino acid peritoneal dialysate; * LBM Lean body mass; * WBPT Whole body protein turnover; * IDPN Intra-dialytic parenteral nutrition; * MICS malnutrition-inflammation complex syndrome; * WBPT Whole body protein synthesis; * UAER Urinary Albumin Excretion Rate; * PCR Protein Catabolic Rate; * nNPA Normalised Protein Appearance; * CRP c-reactive protein; * SGA Subjective Global Assessment; * PG-SGA Patient Generated Subjective Global Assessment; * Hb Haemoglobin; * HDL High density lipoprotein; * LDL low density lipoprotein; *VLDL very low density lipoprotein, TG Triglyceride; * $\mathrm{PO}_{4}$ phosphate; * HCT hydrochlorothiazide; * MUAC Mid Upper Arm Circumference; * TSF Triceps Skinfold Thickness; * MUAMC Mid Upper Arm Muscle Circumference; * MAMC Mid Arm Muscle Circumference; * BMI Body mass index; * EPA Eicosopentanoic Acid; * DHA Decosahexanoic Acid; * Ca Calcium; * ONS Oral Nutrition Support; * CV Cadiovascular; * KDQoL Kidney Disease Quality of Life; * CI Confidence Interval; * Na sodium; * LV left ventricular; * ESRD End Stage Renal Disease; * IQR interquartile range. 
Table 3. Nutritional Parameter in International Guidelines with evidence.

\begin{tabular}{|c|c|c|}
\hline $\begin{array}{l}\text { Nutrient or } \\
\text { Requirement }\end{array}$ & Most Current Equivalent Guideline Statement & $\begin{array}{c}\text { Grade of Evidence } \\
\text { Equivalent to } \\
\text { GRADE [59] } \\
\end{array}$ \\
\hline \multirow{4}{*}{ Energy-dialysis } & KDOQI (2000) [60], BDA (2013) [19] & \multirow[t]{4}{*}{ 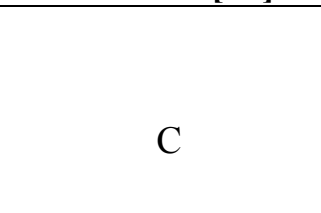 } \\
\hline & The recommended daily energy intake for maintenance haemodialysis or chronic peritoneal dialysis patients is & \\
\hline & $35 \mathrm{kcal} / \mathrm{kg}$ ideal body weight $/$ day $(146 \mathrm{~kJ} / \mathrm{kg}$ IBW/day) for those who are less than 60 years of age and 30 to & \\
\hline & $35 \mathrm{kcal} / \mathrm{kg}$ body weight/day ( $126-146 \mathrm{~kJ} / \mathrm{kg}$ IBW/day) for individuals 60 years or older. & \\
\hline \multirow{4}{*}{ Protein-pre-dialysis } & CARI (2013) [15] & \multirow[b]{2}{*}{$1 \mathrm{C}$} \\
\hline & $\begin{array}{l}\text { We recommend for patients with early CKD consume a normal protein diet of } 0.75-1.0 \mathrm{~g} / \mathrm{kg} \mathrm{IBW} / \text { day with } \\
\text { adequate energy. This is the Recommended Dietary Intake for the general population. }\end{array}$ & \\
\hline & $\begin{array}{l}\text { A low protein diet ( } \leq 0.6 \mathrm{~g} / \mathrm{kg} \text { IBW/day) to slow down CKD progression is not recommended because of the risk } \\
\text { of malnutrition. }\end{array}$ & $1 \mathrm{C}$ \\
\hline & $\begin{array}{l}\text { We suggest that patients with excess protein intakes reduce their intakes to the RDI levels as a high protein diet } \\
\text { may accelerate renal function decline in mild renal insufficiency }\end{array}$ & $2 \mathrm{C}$ \\
\hline
\end{tabular}

\section{ADA (2010) [18]}

For adults with CKD without diabetes, not on dialysis, with an eGFR $<20 \mathrm{~mL} / \mathrm{min}$, a very low protein controlled

Protein-pre-dialysis diet providing $0.3 \mathrm{~g}-0.5 \mathrm{~g}$ dietary protein per kg of body weight per day with addition of keto acid analogs to meet with keto acids protein requirements may be recommended. International studies report that additional keto acid analogs and vitamin or mineral supplementation are needed to maintain adequate nutrition status for patients with CKD who

Strong, conditional evidence consume a very low protein controlled diet $(0.3-0.5 \mathrm{~g} / \mathrm{kg} /$ day $)$ 
Table 3. Cont.

\section{KDOQI (2000) [59] BDA (2013) [19]}

Protein-dialysis The recommended dietary protein intake for clinically and weight stable maintenance HD patients is $1.1 \mathrm{~g} / \mathrm{kg}$ ideal body weight/day. At least $50 \%$ of the dietary protein should be of high biological value. For clinically and weight stable PD patients, the recommended protein intake is $1.0-1.2 \mathrm{~g} / \mathrm{kg}$ ideal body weight/day. Those who are not stable may need higher levels of protein.

CARI (2013) [15]
Sodium-pre-dialysis $\quad$ We recommend that early CKD patients restrict their dietary sodium intake to belo reduces blood pressure and albuminuria in patients with CKD.

Sodium-dialysis Dietary sodium intake of less than $2.4 \mathrm{~g} /$ day (less than $100 \mathrm{mmol} / \mathrm{day}$ ) should be recommended in most adults with CKD and hypertension.

\section{CARI (2013) [15]}

Fluid-pre-dialysis $\quad$ We suggest that patients drink fluids in moderation. For most patients with early CKD, a daily fluid intake of 2-2.5 L (including fluid content of foods) is sufficient, although this may need to be varied for individual circumstances.

\section{CARI (2013) [15]}

We suggest that early CKD patients (stages 1-3) should not restrict dietary phosphate intake as restrictions of dietary phosphate does not influence renal or cardiovascular outcomes in these patients.

\section{KDIGO (2009) [17]}

In patients with CKD stages 3-5, we suggest maintaining serum phosphorus in the normal range.

In patients with CKD stages 3-5 we suggest using phosphate-binding agents in the treatment of hyperphosphatemia.

It is reasonable that the choice of phosphate binder takes into account CKD stage, presence of other components of

CKD-MBD, concomitant therapies, and side-effect profile. 
Table 3. Cont.

\section{KDIGO (2009) [17]}

In patients with CKD stage 5D, we suggest lowering elevated phosphorus levels toward the normal range.

In patients with CKD stages 5D we suggest using phosphate-binding agents in the treatment of hyperphosphatemia.

It is reasonable that the choice of phosphate binder takes into account CKD stage, presence of other components of CKD-MBD, concomitant therapies, and side-effect profile.

In patients with CKD stages 3-5D and hyperphosphatemia, we recommend restricting the dose of calcium-based phosphate binders and/or the dose of calcitriol or vitamin D analog in the presence of persistent

Phosphate-dialysis or recurrent hypercalcemia.

In patients with CKD stages 3-5D and hyperphosphatemia, we suggest restricting the dose of calcium based phosphate binders in the presence of arterial calcification and/or adynamic bone disease and/or if serum PTH levels are persistently low.

In patients with CKD stages 3-5D, we recommend avoiding the long-term use of aluminum-containing phosphate binders and, in patients with CKD stage 5D, avoiding dialysate aluminum contamination to prevent aluminum intoxication.

In patients with CKD stages 3-5D, we suggest limiting dietary phosphate intake in the treatment of hyperphosphatemia alone or in combination with other treatments.

\section{CARI (2103) [15]}

Fibre We suggest patients with early CKD consume a diet rich in dietary fibre that is associated with reduced inflammation and mortality in CKD patients.

\section{CARI (2013) [15]}

Potassium-pre-dialysis We suggest that early CKD patients with persistent hyperkalaemia restrict their dietary potassium intake with the assistance of a qualified dietitian. 
Table 3. Cont

\section{CARI (2013) [15]}

We suggest Vitamin D deficiency ( 25 hydroxy vitamin $\mathrm{D}<37.5 \mathrm{nmol} / \mathrm{L}$ ) and insufficiency ( 25 hydroxy vitamin D $35.5-75 \mathrm{nmol} / \mathrm{L}$ ) if present be corrected using treatment strategies for the general population:

Daily oral intake $19-50$ year: $5 \mu \mathrm{g}$; 51-70 year: $10 \mu \mathrm{g}$; >70 year: $15 \mu \mathrm{g}(1 \mu \mathrm{g}=40 \mathrm{IU})$. It is very difficult to meet RDI with food intake alone.

A few minutes in Australian summer for fair skinned people and $2-3 \mathrm{~h}$ of sunlight/week in winter in southern regions.

Vitamin D-pre-dialysis We recommend a prescription of vitamin D therapy for early CKD patients with secondary hyperparathyroidism, as it has been shown to be effective in suppressing elevated levels of parathryroid (PTH) hormone. There is insufficient evidence to determine whether this improves patient-level outcomes and the potential benefits of vitamin D therapy must be weighed against its potential deleterious effects, including hypercalcaemia, hyperphosphataemia, vascular calcification, adynamic bone disease and accelerated progression of $\mathrm{CKD}$.

We recommend that early CKD patients on vitamin D therapy have their calcium, phosphate, PTH, alkaline phosphate and $25(\mathrm{OH})$ vitamin D level monitored regularly.

\section{KDIGO (2009) [17]}

Vitamin D-dialysis In patients with CKD stage 5D and elevated or rising PTH, we suggest calcitriol, or vitamin D analogs, or calcimimetics, or a combination of calcimimetics and calcitriol or vitamin D analogs be used to lower PTH.

\section{CARI (2013) [15]}

We recommend that overweight/obese patients with CKD should be prescribed caloric restriction under the management of an appropriately qualified dietitian. A reduction in weight can mean an improvement of CKD.

We suggest, in the absence of specific recommendations for CKD, overweight and obese patients are encouraged to aim for a body mass index (BMI) of between 18.5 and $24.9 \mathrm{~kg} / \mathrm{m}^{2}$ and waist circumference of $\leq 102 \mathrm{~cm}$ for men and

Calorie $\leq 88 \mathrm{~cm}$ for women.

restriction/weight loss

\section{CMA (2008) [11]}

Obese (BMI $>30.0 \mathrm{~kg} / \mathrm{m}^{2}$ ) and overweight (BMI 25.0-29.9 kg/m²) people should be encouraged to reduce their BMI to lower their risk of chronic kidney diseaseand end-stage renal disease.

Maintenance of a health body weight (BMI $18.5-24.9 \mathrm{~kg} / \mathrm{m}^{2}$; waistcircumference $<102 \mathrm{~cm}$ for men,

$<88 \mathrm{~cm}$ for women) is recommended to prevent hypertension.

Or to reduce blood pressure in those with hypertension. 
Table 3. Cont.

\section{CARI (2013) [15]}

Other dietary

components

Counselling

Fruit and vegetables - we suggest adults with early CKD consume a balanced diet rich in fruit and vegetables, as these appear to reduce blood pressure and have renoprotective effects comparable to sodium bicarbonate. Mediterranean diet - we suggest adults with CKD consume a Mediterranean style diet to reduce dyslipidemia and to protect against lipid peroxidation and inflammation.

\section{CARI (2013) [15]}

We suggest that patients with progressive CKD have individualised dietary interventions involving an appropriately qualified dietitian.

\section{NICE (2008) [13]}

Where the clinician in discussion with the patient has decided that dietary intervention to influence progression of CKD is indicated, an appropriately trained professional should discuss the risks and benefits of dietary protein restriction, with particular reference to slowing down the progression of disease $v s$. protein-calorie malnutrition.

Where dietary intervention is agreed this should occur within the context of education, detailed dietary assessment and supervision to ensure malnutrition is prevented.

Offer dietary advice to people with progressive CKD concerning potassium, phosphate, protein, calorie and salt intake when indicated.

\section{CMA (2008) [11]}

Renal programs and care providers for patients with progressive chronic kidney disease who choose not to pursue renal replacement therapies should ensure patients have access to an interdisciplinary team to provide

Conservative comprehensive conservative management.

management

- All chronic kidney disease programs and care providers should have a mechanism by which to develop documents and processes for advanced-care planning.

- Comprehensive conservative management protocols should include symptom management, psychological care and spiritual care.

- Coordinated end-of-life care should be available to patients and families. 
The Australian CARI guidelines, shown in Table 3, state that a protein-controlled diet consisting of $0.75-1.0 \mathrm{~g} / \mathrm{kg} /$ day, is recommended for adults pre-dialysis (Stages 3-4) [15]. The administration of a low protein diet $(<0.6 \mathrm{~g} / \mathrm{kg} /$ day $)$ to slow renal failure progression is not justified when the reported clinically modest benefit on glomerular filtration rate decline is weighed against the concomitant significant declines in clinical and biochemical parameters of nutrition [15]. It is the most recent of the international guidelines assessing this question and is at odds with the systematic reviews [22,23].

The British Dietetic Association's guidelines on protein intake in both haemodialysis (HD) and peritoneal dialysis (PD) recommend a lower level of protein intake than previous guidelines at $1.1 \mathrm{~g} / \mathrm{kg}$ ideal body weight/day for those undergoing maintenance haemodialysis and 1.0-1.2 $\mathrm{g} / \mathrm{kg}$ ideal body weight/day for those on maintenance peritoneal dialysis [19]. These recommendations are graded C using the Scottish Intercollegiate Guideline Network criteria, that is based on well-conducted cohort or case control studies with a low risk of confounding and a moderate probability that the relationship is causal [12]. The authors emphasise the importance of adequate energy (126-167 kJ/day in HD and $146 \mathrm{~kJ} /$ day for PD in adults under 60 years and 126-146 kJ/day for those over 60 years). This recommendation is slightly lower than previously recommended and is based on medically well patients with stable body weights and the authors caution when applying these recommendations to less well patients [19].

The guidelines on vitamin D (Table 3) focus on the general population decline in serum 25 hydroxy vitamin D and methods to address this in early CKD (Stages 1-4) [15]. In later stages of disease, recent guidelines focus on the combined effects of calcium, phosphate, parathyroid hormone (PTH) and vitamin D on outcome [14,17]. The cohort study by Wang et al. aimed to explore the relationship between serum 25(OH)-hydroxy vitamin D $(25(\mathrm{OH}) \mathrm{D})$ in $\mathrm{PD}$ patients and long term clinical outcomes [36]. They found that $87 \%$ of the cohort were deficient or insufficient in $25(\mathrm{OH}) \mathrm{D}$ (i.e., $<75 \mathrm{nmol} / \mathrm{L}$ ) and that lower serum 25(OH)D levels were associated with an increased risk of cardiovascular events but not long term mortality [36]. The effects of oral paricalcitol supplementation on biochemical markers (including proteinuria) have been studied in both pre-dialysis and early CKD patients (Stages 1-4). A small, six month randomized controlled trial (RCT) found a modest effect size of oral paricalcitol supplementation of $1 \mu \mathrm{g} /$ day $v s$. placebo, with the intervention group demonstrating a $17.6 \%$ decrease in spot urinary protein-creatinine ratio $v$ s. a $2.9 \%$ increase for controls $(p=0.04)$ [34]. It was also noted in this study that serum iPTH fell significantly amongst those who received paricalcitol supplementation $(p=0.01)$ [34]. Agarwal et al. similarly found that oral paricalcitol supplementation (mean dose $9.5 \mu \mathrm{g} /$ week) was significantly associated with $51 \%$ vs. $25 \%(p=0.004)$ reduction in proteinuria in the intervention group compared to controls and 3.2 greater odds for a reduction in proteinuria independent of treatment for Renal Angiotensin Aldosterone blockade [35]. The KDIGO guidelines (see Table 3) recommend calcitriol or other vitamin therapy in those with elevated parathryroid hormone [17]. The CARI guidelines while recommending vitamin D therapy in early kidney disease for those with elevated PTH warn against the risk of vitamin D therapy in the face of elevated serum calcium and phosphate levels, which should be monitored regularly [15].

The evidence for the modification of fat in CKD to moderate cardiovascular outcomes is limited. Beavers et al. found that supplementation of $6 \mathrm{~g}$ omega-3 fatty acids had no effect on total homocysteine levels in HD patients over 6 months [37]. Saltissi et al. found that dietary compliance was a major issue [38]. A dialysis dietary prescription modified to meet the National Heart Foundation 
guidelines of reduced intake of saturated fat and cholesterol, led to a significant reduction in total cholesterol and low density lipoprotein (LDL) cholesterol in HD patients with little effect in PD patients [38]. All guidelines published since 2006, recommend controlling salt intake below $100 \mathrm{mmol}$ sodium/day (2.3 g sodium) as an important feature of managing hypertension [11,12,15,18], although not addressed at any particular stage of CKD. One randomized controlled double blind crossover study of 34 patients with proteinuria and without diabetes was located reporting the effect of a low sodium diet (50 mmol/day) being as efficacious as treatment with hydrocholorothiazide (an angiotensin receptor II antagonist) at reducing proteinuria and blood pressure when combined with a diuretic [39]. Sodium restriction itself exerted a modest, yet significant, antiproteinuric effect [37]. Actively restricting sodium to less than $100 \mathrm{mmol} /$ day (5 g salt) in those undergoing haemodialysis resulted in less hypertensive medications used ( $7 \%$ vs. 42\%), better ventricular function and less intradialytic hypotension compared to those whose blood pressure was controlled by medication [40]. Using sodium excretion as a surrogate for sodium intake, Boudville showed that excretions in the lowest tertile (114 mmol/day) resulted in significantly fewer hypertensive medications $(2 v s .2 .7, p=0.01)$ used in those with GFR $<30 \mathrm{~mL} / \mathrm{min}$, than those in the highest tertile $(166.7 \mathrm{mmol} /$ day $)$. This effect was even more marked in those with GFR $\leq 15 \mathrm{~mL} / \mathrm{min}$ [41].

The effect of dietary fibre supplements and a high fibre diet, on patient reported symptoms of constipation amongst a PD population, suggested that 6-12 g/day of partially hydrolysed guar gum added to usual intake was as effective as usual laxative treatment for preventing constipation in the majority of included PD patients and was associated with less unfavorable side effects [42]. Both Saltissi and Sutton studies were case series without control groups [38,42].

Compliance with diet prescription remains an issue. Twenty-eight per cent (28\%) of participants under-reported protein intake in both a very low protein diet (VLPD) of $0.3 \mathrm{~g} / \mathrm{kg} /$ day plus keto-acids (KA) diet to a level of $0.66 \mathrm{~g} / \mathrm{kg} /$ day and a low protein diet (LPD) of $0.6 \mathrm{~g} / \mathrm{kg} /$ day in pre-dialysis patients. While compliance was poor in both groups, the prescription of the VLPD + KA delivered improved biochemical markers, with significant improvements noted in serum urea nitrogen, serum bicarbonate and urinary phosphorous [31]. In 423 pre-dialysis patients (Stages 4 and 5) randomized to receive two different protein levels, LPD (0.55 g/kg/day) or a Moderate Protein Diet (MPD) of $0.8 \mathrm{~g} / \mathrm{kg} / \mathrm{day}$, for 3 months with follow up to 48 months there were no differences between groups at 6 and 18 months, however there was greater compliance with the MPD [32]. In a case control study of Stage 3-5 CKD patients, Kanazawa demonstrated the compliant group, with dietary protein intakes maintained at $0.69 \mathrm{~g} / \mathrm{kg}$ body weight/day, had smaller decline in GFR, however no measures of change in body composition were recorded and there was no difference in health-related quality of life [33].

In an open RCT in 28 PD patients, randomized to receive a powdered egg-albumin protein supplement (30 g/day providing $22 \mathrm{~g}$ protein) vs. nutrition counselling over six months resulted in significantly improved serum albumin $(2.64 \pm 0.35$ vs. $3.05 \pm 0.72 \mathrm{~g} / \mathrm{dL})$, energy intake $(1331 \pm 342$ vs. $1872 \pm 698 \mathrm{kcal} /$ day $)$, protein intake $(1.0 \pm 0.3 v s .1 .7 \pm 0.7 \mathrm{~g} / \mathrm{kg})$ and nPNA $(1.00 \pm 0.23 v s .1 .18 \pm$ $0.35 \mathrm{~g} / \mathrm{kg} /$ day) amongst the intervention group when compared to baseline measures, and frequency of moderate-severe malnutrition decreased $28 \%$ in the intervention $v s .6 \%$ in the control group [45]. Interestingly, compliance in this study was reported as $90 \%$.

Teixido-Planas et al. conducted a 12 month open RCT of 65 PD participants, comparing daily consumption of $200 \mathrm{~mL} 1.0 \mathrm{kcal} / \mathrm{mL}$ liquid oral nutrition support (ONS), in addition to usual dietary 
intake, against those who consumed only their usual dietary intake [43]. Based on an "intention to treat" analysis, only an improvement in total lymphocyte count $(p=0.0066)$ between intervention and controls reached significance. The supplement was not found to be suitable for long term use due to non-compliance with $31 \%$ of the intervention group dropping out. A similar study by Caglar et al. with ONS showed improvements in albumin, pre-albumin and SGA [44], however the compliance rate (32\%) was similar, with a $46 \%$ dropout rate.

Five studies investigating the effect of structured dietetic counseling on compliance with dietary prescriptions have shown differing results. Campbell et al. randomized 56 pre-dialysis patients (Stages 4 and 5) to fortnightly, individualized counseling on a prescription of $0.75 \mathrm{~g} / \mathrm{kg} /$ day protein and $145 \mathrm{~kJ} / \mathrm{kg} /$ day energy $v s$. written education material for 3 months [46]. The intervention group had a significantly lower reduction in body cell mass and improvement of $17.7 \mathrm{~kJ} / \mathrm{kg} /$ day energy intake and subjective global assessment (SGA). Improvements in nutritional status in the intervention group translated to significant improvements in the symptoms, cognitive functioning and vitality subscales in the Kidney Disease Quality of Life tool, KDQoL [47]. Sullivan also showed in 279 HD patients in a cluster RCT for 3 months that counseling on reducing phosphates in foods compared to usual care significantly reduced serum phosphate levels by $0.6 \mathrm{mg} / \mathrm{dL}$, largely through improvements in food label reading [48]. Conversely, Morey in $67 \mathrm{HD}$ patients randomized to monthly vs. 6 monthly counseling was unable to maintain a reduction in serum phosphate of $0.25 \mathrm{mg} / \mathrm{dL}$ at 3 months, at the 6 month follow-up [49]. A retrospective cohort study over 2 years of 65 HD patients receiving a 6 monthly dietetic review with intensive follow-up for nutrition parameters falling below recommended levels, showed a significant reduction in malnutrition (SGA-B reducing from $14 \%$ to $3 \%$ ), maintained serum albumin, potassium an dry weight and significant reduction in serum phosphate [50].

The evidence for the effect of nutrients in peritoneal dialysis solution is limited to two small studies [51,52]. Improvements in overall protein balance improved in a randomized cross over study of 8 patients over 14 days [51] and also in another study which was open labeled and not controlled in 16 patients over 3 months [52]. The use of intradialytic parenteral nutrition (IDPN) solution in HD patients has also only been conducted in small studies, showing improvements in hepatic albumin synthesis and whole body fat free mass [53-57]. In the cross over study of 8 patients using both IDPN and oral supplements, the oral administration resulted in persistent anabolic benefits in the post dialysis phase, which was not seen with IDPN [55]. In a case series over 12 months of 24 malnourished PD patients, in which there was significant attrition $>50 \%$, the IDPN was associated with increased body weight and improved serum albumin levels [57]. A small case series in haemodialysis patients, using Percutaneous Endoscopic Gastrostomy feeding showed improvements in anthropometric measures over a 3 month period [58].

\section{Discussion}

The focus of guidelines on nutrition and CKD published since 2006 has been on early prevention and lifestyle modification required to prevent progression to ESRD $[11,12,15]$ or the management of renal bone disease [17]. The KDOQI guidelines on nutrition have not been updated since 2000 [59]. Addressing general population's sub-optimal serum vitamin D levels, as well as in early CKD is a priority. Other chronic diseases, such as obesity, diabetes and hypertension, which affect the population 
at large, require management to prevent progression to CKD [15]. The treatment of these diseases has a large nutrition component which needs to be recognized [6].

The approach for managing elevated serum phosphate, through the use of phosphate binders as an adjunct to the restriction of dietary intake, has also been recognized. The KDIGO guidelines continue to recommend restricting dietary phosphate in combination with other treatments, however the evidence is poor [5]. The CARI guidelines state clearly that restriction of diet runs the risk of precipitating malnutrition and thus has promoted moderate restrictions in protein, phosphate and sodium in the pre-dialysis period to levels commensurate with the general population [15]. The studies, specifically looking at dietitian led control of phosphate intake, showed promise but further studies need to be conducted on the frequency of dietetic counseling to ensure long term impact on dietary control $[49,50]$.

Issues of compliance with restricted diets remains a weakness in many of the studies reported here and those promoting regular contact with a dietitian mostly report improved outcomes. The previous DAA guidelines [7] have had wide currency with dietitians in Australia and New Zealand and are largely still relevant in the present day. These guidelines used the Nutrition Care Process (NCP) to guide the development of clinical questions. The NCP consists of nutrition assessment, diagnosis, intervention and monitoring and evaluation [8] and is outcome driven in that nutritional parameters collected as part of the nutrition assessment and addressed through the nutrition prescription, are then re-assessed or evaluated to establish the impact of the nutrition intervention. These outcomes commonly include intermediate outcomes, such as nutrient intake, anthropometric measures and biochemical markers. Studies on the effect of nutrition prescription on clinical outcomes, such as mortality, hospitalization or cost are limited. While the NCP is useful for practical purposes, grading of evidence in line with international recommendations on harmonizing guidelines is still required [21]. One advantage of these nutrition guidelines is the rigorous independent review process undertaken using the Appraisal of Guidelines for Research and Evaluation (AGREE) tool, which has been recommended for future evaluation of guidelines [60]. Areas requiring most revision for the future include recommendations on vitamin D and phosphate. Further studies on the effect of intradialytic parenteral nutrition and enteral support on dialysis are also warranted.

\section{Conclusions}

Overall, the body of evidence supporting nutritional interventions for improving patient outcomes in CKD is primarily based on low level evidence or isolated randomized clinical trials. Much of the evidence around dietary prescription relies on retrospective and uncontrolled cohort studies and the quality of the body of evidence is poor. Most outcomes assessed are generally biochemical endpoints, such as change in serum levels, rather than clinical ones, such as mortality, hospitalization, cost and patient quality of life. There is general agreement across guideline recommendations for the levels of protein in early CKD and on dialysis; however, guidance on the use of very low protein diets with keto-analogues in conservative treatment of those with GFR $<15 \mathrm{~mL} / \mathrm{min}$ is warranted. While the evidence from a few observational trials suggests that these diets pose no greater risk on mortality than dialysis as treatment, better controlled trials are required to confirm this. Further research on the optimal intakes of sodium, phosphate, fats and fibre in well controlled studies are required, as are studies into micronutrients and other components such as antioxidants. Studies on sun exposure 
combined with diet are required to determine optimal vitamin D status. The collaborative effort to use a global approach to international guidance in management of chronic kidney disease is welcome. While more evidence based studies are warranted, the customary nutrition prescription remains satisfactory with the exception of Vitamin D and phosphate. In these two areas, additional research is urgently needed, given the potential of adverse outcomes for the CKD patient. The role of nutrition in the management of CKD is important and needs to be included in further promotion of research outcomes and future guidelines.

\section{Conflicts of Interest}

The authors declare no conflict of interest.

\section{Acknowledgements}

The authors wish to acknowledge the members of the Dietitians Association Australia (DAA) Renal Guidelines Special Interest Group which contributed to the review of the DAA Evidence Based Guidelines for the Nutritional Management of Chronic Kidney Disease and to thus to this paper. 
Appendix 1. Grading of evidence for different guidelines.

\begin{tabular}{|c|c|c|c|c|c|}
\hline Grading Body & Best evidence (A/1A/Strong) & Good Evidence (B/Fair) & Mixed Evidence (C) & \multicolumn{2}{|c|}{ Weak Evidence (D) } \\
\hline \multirow{3}{*}{$\begin{array}{l}\text { NHMRC. National } \\
\text { Health and Medical } \\
\text { Research Council, } \\
\text { Australia (2009) [20] }\end{array}$} & A-Excellent & B-Good & C-Satisfactory & \multicolumn{2}{|c|}{ D-Poor } \\
\hline & $\begin{array}{l}\text { Body of evidence can be trusted to guide } \\
\text { practice. Several level I or II studies with low } \\
\text { risk of bias; Excellent consistency across } \\
\text { studies; Very large clinical impact; Results } \\
\text { are directly generalisable to target population; } \\
\text { Results are directly applicable to the } \\
\text { Australian healthcare context. }\end{array}$ & $\begin{array}{l}\text { Body of evidence can be trusted to guide } \\
\text { practice in most situations. One or two level II } \\
\text { studies with low risk of bias or systematic } \\
\text { review of multiple level III studies with low } \\
\text { risk of bias. Most studies are consistent and } \\
\text { inconsistencies can be explained. Substantial } \\
\text { clinical impact; Results are directly } \\
\text { generalisable to target population with some } \\
\text { caveats; Results are directly applicable to the } \\
\text { Australian healthcare context with few caveats. }\end{array}$ & $\begin{array}{l}\text { Body of evidence provides some support for } \\
\text { recommendation(s) but care should be taken in its } \\
\text { application. Satisfactory level III studies with low } \\
\text { risk of bias or level I or II studies with moderate } \\
\text { risk of bias. Some inconsistency reflecting genuine } \\
\text { uncertainty around question. Moderate clinical } \\
\text { impact; Not directly generalisable to target } \\
\text { population but could be sensibly applied. Results } \\
\text { are probably applicable to the Australian healthcare } \\
\text { context with some caveats. }\end{array}$ & \multicolumn{2}{|c|}{$\begin{array}{l}\text { Body of evidence is weak and } \\
\text { recommendation must be applied with } \\
\text { caution. Level IV studies or level I to III } \\
\text { studies with high risk of bias. Evidence is } \\
\text { inconsistent; Slight or restricted clinical } \\
\text { impact. Not directly generalisable to target } \\
\text { population hard to judge whether it is } \\
\text { sensible to apply. Results are not } \\
\text { applicable to the Australian healthcare } \\
\text { context. }\end{array}$} \\
\hline & $\mathbf{A}$ & B & $\mathbf{C}$ & D & $\begin{array}{c}\text { Good Practice } \\
\text { Points } \\
\end{array}$ \\
\hline $\begin{array}{l}\text { SIGN Scottish } \\
\text { Intercol-legiate } \\
\text { Guidelines Network } \\
2008[12]\end{array}$ & $\begin{array}{l}\text { At least one meta-analysis, systematic } \\
\text { review, or RCT rated as 1++, and directly } \\
\text { applicable to the target population; OA body } \\
\text { of evidence consisting principally of studies } \\
\text { rated as 1+, directly applicable to the target } \\
\text { population, and demonstrating overall } \\
\text { consistency of results. }\end{array}$ & $\begin{array}{l}\text { A body of evidence including studies rated as } \\
2++ \text {, directly applicable to the target } \\
\text { population, and demonstrating overall } \\
\text { consistency of results; OR; Extrapolated } \\
\text { evidence from studies rated as } 1++ \text { or } 1+\text {. }\end{array}$ & $\begin{array}{l}\text { A body of evidence including studies rated as } 2+\text {, } \\
\text { directly applicable to the target population, and } \\
\text { demonstrating overall consistency of results; OR; } \\
\text { Extrapolated evidence from studies rated as } 2++ \text {. }\end{array}$ & $\begin{array}{l}\text { Evidence level } 3 \text { or } \\
4 \text {; Extrapolated } \\
\text { evidence from } \\
\text { studies rated } \\
\text { as } 2+\text {. }\end{array}$ & $\begin{array}{l}\text { Recommended } \\
\text { best practice based } \\
\text { on the clinical } \\
\text { experience of the } \\
\text { guidelines } \\
\text { development } \\
\text { group. }\end{array}$ \\
\hline
\end{tabular}




\section{Appendix 1. Cont.}

Canadian Society Nephrology (2008) [11]

High quality RCT or meta-analyses with adequate power and clinically important outcomes.
High quality RCT or meta-analyses with

adequate power but outcome is a validated surrogate or results need to be extrapolated from study population to real population OR; High quality RCT or meta-analyse with inadequate power but with clinically important or validated surrogate outcome
High quality RCT or

meta-analyses with adequate

power but outcome is neither

clinically important or a validated

surrogate outcome OR;

Observational study with

statistically significant results

and outcome is clinically

important or a validated

surrogate AND study population

is representative of population

recommendation is for OR

results can be extrapolated from

study population to

real population.

\section{A High}

We are confident that the true effect lies close to that of the estimate of the effect.

\section{KDIGO}

Kidney Disease

Improving Global

Outcomes (2013) [5]

\section{Level 1 "We recommend".}

Most people in situation would want the

recommended course of action and only a

small proportion would not. The

recommendation can be evaluated as a

candidate for developing a policy or a

performance measure.

\section{B Moderate}

C Low

D Very Low

The true effect is likely to be close to the

estimate of the effect, but there is a possibility that it is substantially different. Level 2 "We suggest"; The majority of people in situation would want the

recommended course of action, but many would not. The recommendation is likely to require substantial debate and

involvement of stakeholders before policy

can be determined.
The true effect may be substantially different from the estimate of the effect.

High quality RCT or meta-analyses with inadequate power and neither clinically important nor validated surrogate outcomes OR; Observational study with statistically significant results but neither clinically important nor validated surrogate outcome OR;

Observational study with inadequate power and applicability of the study is irrelevant.

The estimate of effect is very uncertain, and often will be far from the truth. 


\section{Appendix 1. Cont.}

C

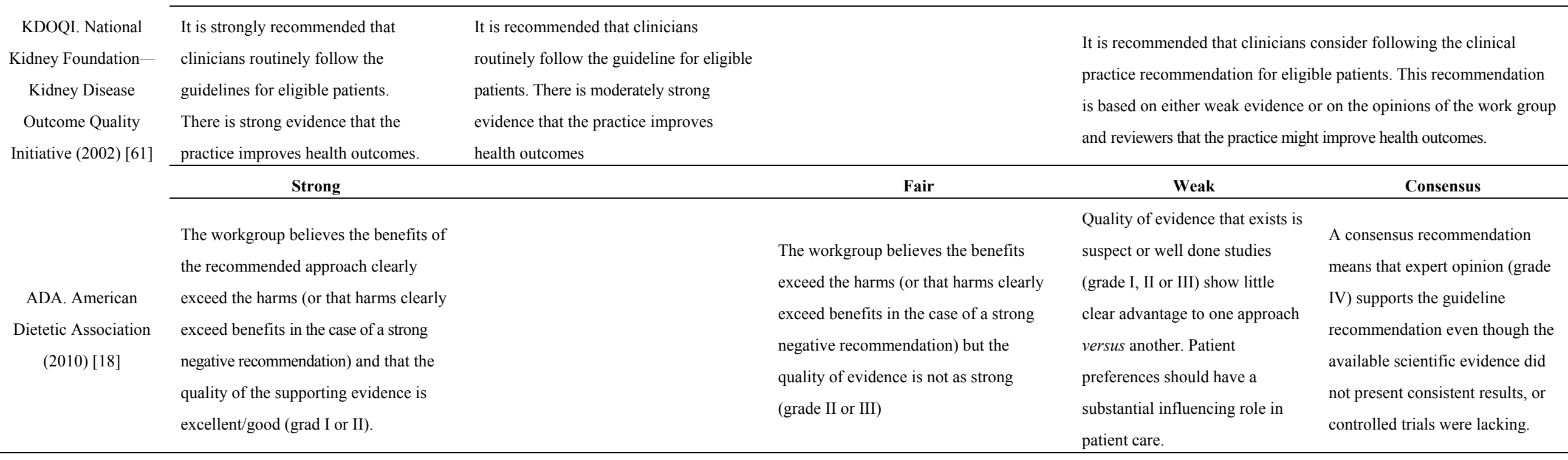


Appendix 1. Cont.

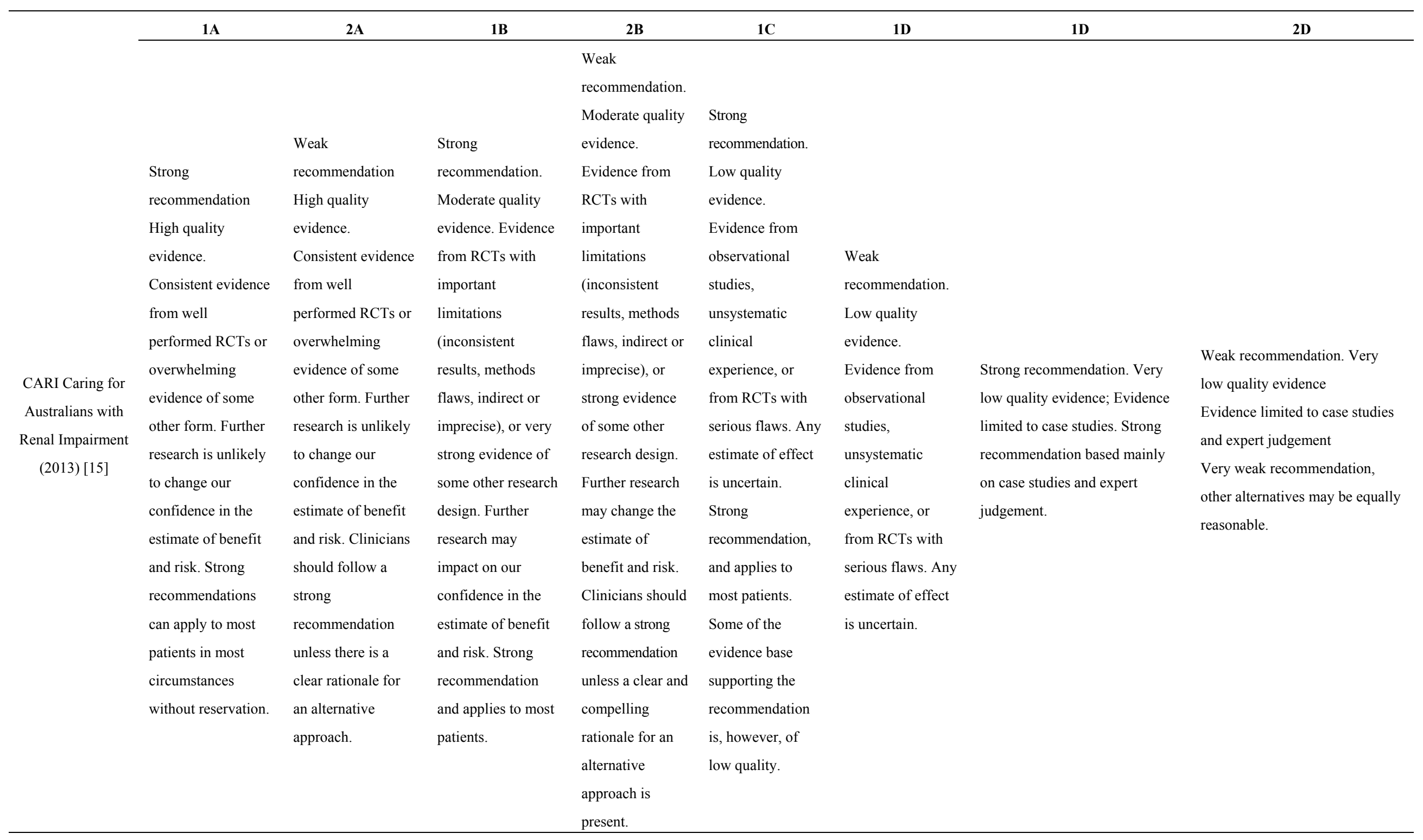




\section{Appendix 1. Cont.}

High

Grading of

Recommendations

Assessment

Development

Evaluation

(GRADE) [59]
We are very confident that the true effect lies close to that of the estimate of the effect. Further research is very unlikely to change our confidence in the estimate of effect.
Moderate

We are moderately confident in the effect estimate: The true effect is likely to be close to the estimate of the effect, but there is a possibility that it is

substantially different. Further research is likely to have an important impact on our confidence in the estimate of effect and may change the estimate.
Low

Very low

Our confidence in the effect estimate is limited: The true effect may be

substantially different from the estimate of the effect. Further research is very

We have very little confidence in the effect estimate: The true effect is likely to be substantially different from the estimate of effect. Any likely to have an important impact on our estimate of effect is very uncertain. confidence in the estimate of effect and is likely to change the estimate. 


\section{References}

1. Australian Institute of Health and Welfare. Projections of the Incidence of Treated End-Stage Kidney Disease in Australia; AIHW: Canberra, Australia, 2011.

2. Weiner, D.E.; Tighiouart, H.; Amin, M.G.; Stark, P.C.; MacLeod, B.; Griffith, J.L.; Salem, D.N.; Levey, A.S.; Sarnak, M.J. Chronic kidney disease as a risk factor for cardiovascular disease and all-cause mortality: A pooled analysis of community-based studies. J. Am. Soc. Nephrol. 2004, $15,1307-1315$.

3. Muscaritoli, M.; Molfino, A.; Bollea, M.R.; Fanelli, F.R. Malnutrition and wasting in renal disease. Curr. Opin. Clin. Nutr. Metab. Care 2009, 12, 378-383.

4. Cass, A.; Chadban, S.; Gallagher, M.; Howard, K.; Jones, A.; McDonald, S.; Snelling, P.; White, S. The Economic Impact of End-Stage Kidney Disease in Australia: Projections to 2020; Kidney Health Australia: Canberra, Australia, 2010.

5. KDIGO Kidney Disease: Improving Global Outcomes CKD Work Group. KDIGO 2012 clinical practice guideline for the evaluation and management of chronic kidney disease. Kidney Int. Suppl. 2013, 3, 1-150.

6. Levey, A.S.; Schoolwerth, A.C.; Burrows, N.R.; Williams, D.E.; Stith, K.R.; McClellan, W. Comprehensive public health strategies for preventing the development, progression, and complications of CKD: Report of an expert panel convened by the Centers for Disease Control and Prevention. Am. J. Kidney Dis. 2009, 53, 522-535.

7. Ash, S.; Campbell, K.; MacLaughlin, H.; McCoy, E.; Chan, M.; Anderson, K.; Corke, K.; Dumont, R.; Lloyd, L.; Meade, A.; et al. Evidence based practice guidelines for the nutritional management of chronic kidney disease. Nutr. Diet. 2006, 63, S35-S45.

8. Lacey, K.; Pritchett, E. Nutrition care process and model: ADA adopts road map to quality care and outcomes management. J. Am. Diet. Assoc. 2003, 103, 1061-1072.

9. Brozek, J.L.; Akl, E.A.; Alonso-Coello, P.; Lang, D.; Jaeschke, R.; Williams, J.W.; Phillips, B.; Lelgemann, M.; Lethaby, A.; Bousquet, J.; et al. Grading quality of evidence and strength of recommendations in clinical practice guidelines. Part 1 of 3 . An overview of the GRADE approach and grading quality of evidence about interventions. Allergy 2009, 64, 669-677.

10. Fouque, D.; Vennegoor, M.; Ter Wee, P.; Wanner, C.; Basci, A.; Canaud, B.; Haage, P.; Konner, K.; Kooman, J.; Martin-Malo, A.; et al. EBPG Guideline on Nutrition. Nephrol. Dial. Transpl. 2007, 22, ii45-ii87.

11. Levin, A.; Hemmelgarn, B.; Culleton, B.; Tobe, S.; McFarlane, P.; Ruzicka, M.; Burns, K.; Manns, B.; White, C.; Madore, F.; et al. Guidelines for the management of chronic kidney disease. Can. Med. Assoc. J. 2008, 179, 1154.

12. Scottish Intercollegiate Guidelines Network. Diagnosis and Management of Chronic Kidney Disease: A National Clinical Guideline; Elliott House: Edinburgh, UK, 2008.

13. National Collaborating Centre for Chronic Conditions. Chronic Kidney Disease: National Clinical Guideline for Early Identification and Management in Adults in Primary and Secondary Care; Royal College of Physicians: London, UK, 2008.

14. Elder, G.; Faull, R.; Branley, P.; Hawley, C. The CARI guidelines. Management of bone disease, calcium, phosphate and parathyroid hormone. Nephrology 2006, 11, S230-S261. 
15. Johnson, D.W.; Atai, E.; Chan, M.; Phoon, R.K.S.; Scott, C.; Toussaint, N.D.; Turner, G.L.; Usherwood, T.; Wiggins, K.J. KHA-CARI guideline: Early chronic kidney disease: Detection, prevention and management. Nephrology 2013, 18, 340-350.

16. Pollock, C.; Voss, D.; Hodson, E.; Crompton, C. The CARI Guidelines. Nutrition and growth in kidney disease. Nephrology 2005, 10, S177-S230.

17. KDIGO Kidney Disease: Improving Global Outcomes (KDIGO) CKD-MBD Work Group. KDIGO clinical practice guideline for the diagnosis, evaluation, prevention, and treatment of chronic kidney disease-mineral and bone disorder (CKD-MBD). Kidney Int. 2009, 113, S1-S130.

18. Clearinghouse, N.G. Chronic Kidney Disease Evidence-Based Nutrition Practice Guideline. Available online: http://www.guideline.gov (accessed on 15 January 2014).

19. Naylor, H.L.; Jackson, H.; Walker, G.H.; Macafee, S.; Magee, K.; Hooper, L.; Stewart, L.; MacLaughlin, H.L.; Renal Nutrition Group of the British Dietetic Association. British Dietetic Association evidence-based guidelines for the protein requirements of adults undergoing maintenance haemodialysis or peritoneal dialysis. J. Hum. Nutr. Diet. 2013, 26, 315-328.

20. National Health and Medical Research Council. NHMRC Levels of Evidence and Grades for Recommendations for Developers of Guidelines; Australian Government: Canberra, Australia, 2009.

21. Vanbelleghem, H.V.R.; Levin, N.W.; Becker, G.; Craig, J.C.; Ito, S.; Lau, J.; Locatelli, F.; Zoccali, C.; Solez, K.; Hales, M.; et al. The kidney disease: Improving global outcomes website: Comparison of guidelines as a tool for hamronisation. Kidney Int. 2007, 71, 1054-1061.

22. Fouque, D.; Laville, M. Low protein diets for chronic kidney disease in non diabetic adults. Cochrane Database Syst. Rev. 2006, doi: 10.1002/14651858.CD001892.pub2.

23. Zarazaga, A.; Garcia-De-Lorenzo, L.; Garcia-Luna, P.P.; Garcia-Peris, P.; Lopez-Martinez, J.; Lorenzo, V.; Quecedo, L.; Del Llano, J. Nutritional support in chronic renal failure: Systematic review. Clin. Nutr. 2001, 20, 291-299.

24. Robertson, L.M.; Waugh, N.; Robertson, A. Protein restriction for diabetic renal disease. Cochrane Database Syst. Rev. 2007, doi: 10.1002/14651858.CD002181.pub2.

25. Navaneethan, S.D.; Yehnert, H.; Moustarah, F.; Schreiber, M.J.; Schauer, P.R.; Beddhu, S. Weight loss interventions in chronic kidney disease: A systematic review and meta-analysis. Clin. J. Am. Soc. Nephrol. 2009, 4, 1565-1574.

26. Stratton, R.J.; Bircher, G.; Fouque, D.; Stenvinkel, P.; de Mutsert, R.; Engfer, M.; Elia, M. Multinutrient oral supplements and tube feeding in maintenance dialysis: A systematic review and meta-analysis. Am. J. Kidney Dis. 2005, 46, 387-405.

27. Chauveau, P.; Couzi, L.; Vendrely, B.; de Precigout, V.; Combe, C.; Fouque, D.; Aparicio, M. Long-term outcome on renal replacement therapy in patients who previously received a keto acid-supplemented very-low-protein diet. Am. J. Clin. Nutr. 2009, 90, 969-974.

28. Brunori, G.; Viola, B.F.; Parrinello, G.; de Biase, V.; Como, G.; Franco, V.; Garibotto, G.; Zubani, R.; Cancarini, G.C. Efficacy and safety of a very-low-protein diet when postponing dialysis in the elderly: A prospective randomized multicenter controlled study. Am. J. Kidney Dis. 2007, 49, 569-580.

29. Vendrely, B.; Chauveau, P.; Barthe, N.; El Haggan, W.; Castaing, F.; de Precigout, V.; Combe, C.; Aparicio, M. Nutrition in hemodialysis patients previously on a supplemented very low protein diet. Kidney Int. 2003, 63, 1491-1498. 
30. Shinaberger, C.S.; Kilpatrick, R.D.; Regidor, D.L.; McAllister, C.J.; Greenland, S.; Kopple, J.D.; Kalantar-Zadeh, K. Longitudinal associations between dietary protein intake and survival in hemodialysis patients. Am. J. Kidney Dis. 2006, 48, 37-49.

31. Feiten, S.F.; Draibe, S.A.; Watanabe, R.; Duenhas, M.R.; Baxmann, A.C.; Nerbass, F.B.; Cuppari, L. Short-term effects of a very-low-protein diet supplemented with ketoacids in nondialyzed chronic kidney disease patients. Eur. J. Clin. Nutr. 2005, 59, 129-136.

32. Cianciaruso, B.; Pota, A.; Bellizzi, V.; Giuseppe, D.D.; di Micco, L.; Minutolo, R.; Pisani, A.; Sabbatini, M.; Ravani, P. Effect of a low-versus moderate-protein diet on progression of CKD: Follow-up of a randomized controlled trial. Am. J. Kidney Dis. 2009, 54, 1052-1061.

33. Kanazawa, Y.; Nakao, T.; Ohya, Y.; Shimomitsu, T. Association of socio-psychological factors with the effects of low protein diet for the prevention of the progression of chronic renal failure. Intern. Med. 2006, 45, 199-206.

34. Fishbane, S.; Chittineni, H.; Packman, M.; Dutka, P.; Ali, N.; Durie, N. Oral paricalcitol in the treatment of patients with CKD and proteinuria: A randomized trial. Am. J. Kidney Dis. 2009, 54, $647-652$.

35. Agarwal, R.; Rizkala, A.R.; Bastani, B.; Kaskas, M.O.; Leehey, D.J.; Besarab, A. A randomized controlled trial of oral versus intravenous iron in chronic kidney disease. Am. J. Nephrol. 2006, $26,445-454$.

36. Wang, A.Y.M.; Lam, C.W.K.; Sanderson, J.E.; Wang, M.; Chan, I.H.S.; Lui, S.F.; Sea, M.M.M.; Woo, J. Serum 25-hydroxyvitamin D status and cardiovascular outcomes in chronic peritoneal dialysis patients: A 3-year prospective cohort study. Am. J. Clin. Nutr. 2008, 87, 1631-1638.

37. Beavers, K.M.; Beavers, D.P.; Bowden, R.G.; Wilson, R.L.; Gentile, M. Omega-3 fatty acid supplementation and total homocysteine levels in end-stage renal disease patients. Nephrology 2008, 13, 284-288.

38. Saltissi, D.; Morgan, C.; Knight, B.; Chang, W.; Rigby, R.; Westhuyzen, J. Effect of lipid-lowering dietary recommendations on the nutritional intake and lipid profiles of chronic peritoneal dialysis and hemodialysis patients. Am. J. Kidney Dis. 2001, 37, 1209-1215.

39. Vogt, L.; Waanders, F.; Boomsma, F.; de Zeeuw, D.; Navis, G. Effects of dietary sodium and hydrochlorothiazide on the antiproteinuric efficacy of losartan. J. Am. Soc. Nephrol. 2008, 19, 999-1007.

40. Kayikcioglu, M.; Tumuklu, M.; Ozkahya, M.; Ozdogan, O.; Asci, G.; Duman, S.; Toz, H.; Can, L.H.; Basci, A.; Ok, E. The benefit of salt restriction in the treatment of end-stage renal disease by haemodialysis. Nephrol. Dial. Transpl. 2009, 24, 956-962.

41. Boudville, N.; Ward, S.; Benaroia, M.; House, A.A. Increased sodium intake correlates with greater use of antihypertensive agents by subjects with chronic kidney disease. Am. J. Hypertens. 2005, 18, 1300-1305.

42. Sutton, D.; Dumbleton, S.; Allaway, C. Can increased dietary fibre reduce laxative requirement in peritoneal dialysis patients? J. Renal Care 2007, 33, 174-178.

43. Teixido-Planas, J.; Ortiz, A.; Coronel, F.; Montenegro, J.; Lopez-Menchero, R.; Ortiz, R.; Gomez, C.; Donate, T. Oral protein-energy supplements in peritoneal dialysis: A multicenter study. Perit. Dial. Int. 2005, 25, 163-172. 
44. Caglar, K.; Fedje, L.; Dimmitt, R.; Hakim, R.M.; Shyr, Y.; Ikizler, T.A. Therapeutic effects of oral nutritional supplementation during hemodialysis. Kidney Int. 2002, 62, 1054-1059.

45. Gonzalez-Espinoza, L.; Gutierrez-Chavez, J.; Martin del Campo, F.; Martinez-Ramirez, H.R.; Cortes-Sanabria, L.; Rojas-Campos, E.; Cueto-Manzano, A.M. Randomized, open label, controlled clinical trial of oral administration of an egg albumin-based protein supplement to patients on continuous ambulatory peritoneal dialysis. Perit. Dial. Int. 2005, 25, 173-180.

46. Campbell, K.; Ash, S.; Davies, P.; Bauer, J. Randomized controlled trial of nutritional counseling on body composition and dietary intake in severe CKD. Am. J. Kidney Dis. 2008, 51, 748-758.

47. Campbell, K.L.; Ash, S.; Bauer, J.D. The impact of nutrition intervention on quality of life in pre-dialysis chronic kidney disease patients. Clin. Nutr. 2008, 27, 537-544.

48. Sullivan, C.; Sayre, S.S.; Leon, J.B.; Machekano, R.; Love, T.E.; Porter, D.; Marbury, M.; Sehgal, A.R. Effect of food additives on hyperphosphatemia among patients with end-stage renal disease: A randomized controlled trial. JAMA 2009, 301, 629-635.

49. Morey, B.; Walker, R.; Davenport, A. More dietetic time, better outcome? A randomized prospective study investigating the effect of more dietetic time on phosphate control in end-stage kidney failure haemodialysis patients. Nephron Clin. Pract. 2008, 109, c173-c180.

50. Campbell, K.L.; Ash, S.; Zabel, R.; McFarlane, C.; Juffs, P.; Bauer, J.D. Implementation of standardized nutrition guidelines by renal dietitians is associated with improved nutrition status. J. Renal Nutr. 2009, 19, 136-144.

51. Tjiong, H.L.; van den Berg, J.W.; Wattimena, J.L.; Rietveld, T.; van Dijk, L.J.; van der Wiel, A.M.; van Egmond, A.M.; Fieren, M.W.; Swart, R. Dialysate as food: Combined amino acid and glucose dialysate improves protein anabolism in renal failure patients on automated peritoneal dialysis. J. Am. Soc. Nephrol. 2005, 16, 1486-1493.

52. Sezer, M.T.; Demir, M.; Ertürk, J.; Yildiz, M. Effects of amino acid peritoneal dialysate in malnourished peritoneal dialysis patients. Eur. J. Gen. Med. 2006, 3, 58-63.

53. Pupim, L.B.; Flakoll, P.J.; Ikizler, T.A. Nutritional supplementation acutely increases albumin fractional synthetic rate in chronic hemodialysis patients. J. Am. Soc. Nephrol. 2004, 15, 1920-1926.

54. Pupim, L.B.; Majchrzak, K.M.; Flakoll, P.J.; Ikizler, T.A. Intradialytic oral nutrition improves protein homeostasis in chronic hemodialysis patients with deranged nutritional status. J. Am. Soc. Nephrol. 2006, 17, 3149-3157.

55. Cherry, N.; Shalansky, K. Efficacy of intradialytic parenteral nutrition in malnourished hemodialysis patients. Am. J. Health-Syst. Pharm. 2002, 59, 1736-1741.

56. Joannidis, M.; Rauchenzauner, M.; Leiner, B.; Rosenkranz, A.; Ebenbichler, C.F.; Laimer, M.; Tatarczyk, T.; Meusburger, E.; Mayer, G. Effect of intradialytic parenteral nutrition in patients with malnutrition-inflammation complex syndrome on body weight, inflammation, serum lipids and adipocytokines: Results from a pilot study. Eur. J. Clin. Nutr. 2008, 62, 789-795.

57. Korzets, A.; Azoulay, O.; Ori, Y.; Zevin, D.; Boaz, M.; Herman, M.; Chagnac, A.; Gafter, U. The use of intradialytic parenteral nutrition in acutely ill haemodialysed patients. J. Renal Care $\mathbf{2 0 0 8 ,}$ $34,14-18$.

58. Sayce, H.A.; Rowe, P.A.; McGonigle, R.J.S. Percutaneous endoscopic gastrostomy feeding in haemodialysis out-patients. J. Hum. Nutr. Diet. 2000, 13, 333-341. 
59. National Kidney Foundation. Clinical practice guidelines for nutrition in chronic renal failure. Am. J. Kidney Dis. 2000, 35, S1-S140.

60. Brozek, J.L.; Akl, E.A.; Compalati, E.; Kreis, J.; Terracciano, L.; Fiocchi, A.; Ueffing, E.; Andrews, J.; Alonso-Coello, P.; Meerpohl, J.J.; et al. Grading quality of evidence and strength of recommendations in clinical practice guidelines part 3 of 3 . The GRADE approach to developing recommendations. Allergy 2011, 66, 588-595.

61. National Kidney Foundation. K/DOQI clinical practice guidelines for chronic kidney disease: Evaluation, classification, and stratification. Am. J. Kidney Dis. 2002, 39, S1-S266.

(C) 2014 by the authors; licensee MDPI, Basel, Switzerland. This article is an open access article distributed under the terms and conditions of the Creative Commons Attribution license (http://creativecommons.org/Licenses/by/3.0/). 